TRANSACTIONS OF THE

AMERICAN MATHEMATICAL SOCIETY

Volume 361, Number 8, August 2009, Pages 4367-4403

S 0002-9947(09)04833-8

Article electronically published on March 4, 2009

\title{
FLOW INVARIANCE FOR NONLINEAR PARTIAL DIFFERENTIAL DELAY EQUATIONS
}

\author{
WOLFGANG M. RUESS
}

\begin{abstract}
Several fundamental results on existence, flow-invariance, regularity, and linearized stability of solutions to the nonlinear partial differential delay equation $\dot{u}(t)+B u(t) \ni F\left(u_{t}\right), t \geq 0, u_{0}=\varphi$, with $B \subset X \times X$ $\omega$-accretive, are developed for a general Banach space $X$. In contrast to existing results, with the history-response $F$ globally defined and, at least, Lipschitz on bounded sets, the results are tailored for situations with $F$ defined on (possibly) thin subsets of the initial-history space $E$ only, and are applied to place several classes of population models in their natural $L^{1}$-setting. The main result solves the open problem of a subtangential condition for flow-invariance of solutions in the fully nonlinear case, paralleling those known for the cases of (a) no delay, (b) ordinary delay equations with $B \equiv 0$, and (c) the semilinear case.
\end{abstract}

\section{INTRODUCTION}

The object of study of this paper is the following partial functional differential equation with delay:

$$
(\mathrm{PFDE})\left\{\begin{array}{l}
\dot{u}(t)+B u(t) \ni F\left(u_{t}\right), \quad t \geq 0, \\
u_{\mid I}=\varphi \in \hat{E}
\end{array}\right.
$$

$B \subset X \times X$ is a (generally) nonlinear and multivalued operator in a Banach (state) space $X$, with $(B+\omega I)$ accretive, some $\omega \in \mathbb{R}$, and, for given $I=[-R, 0], R>0$ (finite delay), or $I=(-\infty, 0]$ (infinite delay), and $t \geq 0, u_{t}: I \rightarrow X$ is the history of $u$ up to $t: u_{t}(s)=u(t+s), s \in I$, and $\varphi: I \rightarrow X$ is a given initial history out of a subset $\hat{E}$ of a space $E$ of functions from $I$ to $X$. Moreover, $F$ is a given history-responsive operator with domain $D(F) \subset E$ and range in $X$. Usually, $B$ is a (possibly perturbed) partial differential operator in $X$, and equation (PFDE) is used to model evolutionary processes where the time-rate of change of the process depends (not only on the current state, but also) on the past history of the process, such as processes with thermal or shape memory in physics, chemical reaction sequences, and, most notably, population models.

One of the characteristic features of the existing literature on (PFDE) (compare the references below, or, for a rather complete survey, cf. [65] and the references therein) is the assumption on the history-responsive operator $F$ to be globally

Received by the editors October 2, 2007.

2000 Mathematics Subject Classification. Primary 47J35, 35R10; Secondary 47H06, 47H20, 47N60, 92D25.

Key words and phrases. Nonlinear partial differential delay equations, accretive operators, flow-invariance, linearized stability, regularity, population models.

(C)2009 American Mathematical Society Reverts to public domain 28 years from publication 
defined and globally Lipschitz, or, at least, Lipschitz on bounded sets. For applications, this can turn out to be a severe restriction. Some of the prominent examples to this effect are population models, such as the following example modeling the production of red blood cells (for details and further classes of examples, see section $5)$ :

$$
\left\{\begin{array}{l}
\frac{\partial}{\partial t} u(x, t)-d \Delta u(x, t)=a u(x, t)\left[1-b u(x, t)-\int_{-R}^{0} u(x, t+s) d \eta(s)\right] \\
u_{\mid[-R, 0]}=\varphi \in C([-R, 0], X) \\
+ \text { boundary conditions. }
\end{array}\right.
$$

Due to the quadratic terms in the history-response, and thus, for having $F$ globally defined, the problem of invariance of the state space under products, in the existing literature on models of this type, the state space $X$ is taken to be either $C(\bar{\Omega})$ or $W^{2, p}(\Omega), p>N$, with $\Omega$ open and bounded in $\mathbb{R}^{N}$. With $u(t, \cdot)$ representing a population density, however, the natural state space would be $L^{1}(\Omega)$. While, for this state space, the history-response $F(\varphi)=a \varphi(0)\left[1-b \varphi(0)-\int_{-R}^{0} \varphi(s) d \eta(s)\right]$ is not even defined on any subset with an interior point, much less globally, it is, in turn, well-defined on the subset $\hat{E}$ of all $\varphi \in C\left([-R, 0], L^{1}(\Omega)\right)$ for which $\varphi(s) \geq 0, s \in[-R, 0]$, and $\varphi(0) \in L^{\infty}(\Omega)$ - certainly a reasonable restriction on population densities. Obviously, in order to solve problem (1.1) in the $L^{1}-$ context, a corresponding flow-invariance of the solution $u$ is required.

In order to enlarge the range of applicability in general, we provide in this paper a solution theory with flow-invariance for history-responses $F$ defined (possibly) only on thin subsets of $E$. In particular, we extend the corresponding first attempts of [67, 70] (with range assumptions, see Lemma 2.7 below) to the "usual" subtangential assumptions.

Subtangential conditions for flow-invariance are well known for the special cases (a) of no delay (cf. 22, 42, Ch. VIII], [54, 56, 60, and, for the most recent account as well as further references, [9, 10]), and (b) of ordinary functional differential equations with $B \equiv 0($ 39, 40, 41, 75]), while (c) the semilinear case of (PFDE), with $B: D(B) \subset X \rightarrow X$ (single-valued and) linear, and $-B$ generating a $C_{0}-$ semigroup of bounded linear operators on $X$, has been solved in 43, 45, 55, 56. (For a rather complete account of the semilinear case of (PFDE) in general, we refer to [87].)

A corresponding result for the general nonlinear case is a long-standing open problem. The main result of this paper (Theorem 2.1 below) provides a solution to this problem by a sufficient subtangential condition for flow-invariance of solutions to (PFDE).

The paper is organized as follows: The principal results for existence and flowinvariance for solutions to (PFDE) are presented in section 2; their proofs are given in section 3. Section 4 is devoted to regularity and linearized stability of solutions. In section 5, the abstract results are applied to models from population dynamics.

Notation and terminology. Throughout this paper, all Banach spaces are assumed to be over the reals. For $X$ a Banach space, $X^{*}$ denotes the (continuous) dual space of $X$, and the action of $x^{*} \in X^{*}$ at $x \in X$ is denoted by $\left\langle x^{*}, x\right\rangle:=x^{*}(x)$. The duality map $J: X \rightarrow 2^{X^{*}}$ is defined by

$$
J(x)=\left\{x^{*} \in X^{*} \mid\left\langle x^{*}, x\right\rangle=\|x\| \text {, and }\left\|x^{*}\right\| \leq 1\right\}, \quad x \in X .
$$


For $\lambda \in \mathbb{R} \backslash\{0\}$, define $[\cdot, \cdot]_{\lambda}: X \times X \rightarrow \mathbb{R}$ by

$$
[x, y]_{\lambda}=\frac{\|x+\lambda y\|-\|x\|}{\lambda} .
$$

Then, as, for fixed $x, y \in X$, the function $\left\{\lambda \rightarrow[x, y]_{\lambda}\right\}$ is nondecreasing for $\lambda>0$, one can define the bracket $[x, y]$ (the right-hand Gâteaux-derivative of the norm at $x$ in the direction of $y$ ) by

$$
[x, y]=\lim _{\lambda \searrow 0}[x, y]_{\lambda}=\inf _{\lambda>0}[x, y]_{\lambda} .
$$

We note that, for $x, y \in X,[x, y]=\max \left\{\left\langle x^{*}, y\right\rangle \mid x^{*} \in J(x)\right\}$ (cf. [7, 48]).

Given a subset $D$ of $X, \mathrm{cl} D$ will denote its closure in $X$. Recall that a subset $C \subset X \times X$ is said to be accretive in $X$ if for each $\lambda>0$ and each pair $\left(x_{i}, y_{i}\right) \in$ $C, i \in\{1,2\}$, we have $\left\|\left(x_{1}+\lambda y_{1}\right)-\left(x_{2}+\lambda y_{2}\right)\right\| \geq\left\|x_{1}-x_{2}\right\|$, and $\omega$-accretive in $X$ for some $\omega \in \mathbb{R}$, if $(C+\omega I)$ is accretive. If, in addition, $R(I+\lambda C)=X$ for all $\lambda>0$ with $\lambda \omega<1$, then $C$ is said to be $\omega$-m-accretive. If $C \subset X \times X$ is $\omega$-accretive, then, for any $\lambda>0$ with $\lambda \omega<1, J_{\lambda}^{C}=(I+\lambda C)^{-1}$ denotes the resolvent of $C$.

Throughout the paper, we shall make use of the following reformulations of $\omega$ accretivity: For a subset $C \subset X \times X$, the following are equivalent:

(a) $C$ is $\omega$-accretive;

(b) $\left\|J_{\lambda}{ }^{C} x_{1}-J_{\lambda}{ }^{C} x_{2}\right\| \leq(1-\lambda \omega)^{-1}\left\|x_{1}-x_{2}\right\|$ for all $x_{1}, x_{2} \in R(I+\lambda C)$, and all $\lambda>0$ with $\lambda \omega<1$;

(c) for all $\left(x_{1}, y_{1}\right),\left(x_{2}, y_{2}\right) \in C$, there exists $x^{*} \in J\left(x_{1}-x_{2}\right)$ such that $-\omega\left\|x_{1}-x_{2}\right\|$ $\leq\left\langle x^{*}, y_{1}-y_{2}\right\rangle$.

Notions of solutions. Given an initial-history space $E$ of functions from $I$ to $X, T>0$, and $B \subset X \times X \omega$-accretive for some $\omega \in \mathbb{R}$, a function $u: I \cup[0, T] \rightarrow X$ will be called a strong, or mild solution to (PFDE) if (i) $u_{\mid I}=\varphi$, and (ii) $u_{\mid[0, T]}$ is continuous and a solution of the corresponding type to the initial-value problem

$$
(\mathrm{CP})\left\{\begin{array}{l}
\dot{u}(t)+B u(t) \ni f(t), \quad 0 \leq t \leq T, \\
u(0)=\varphi(0)
\end{array}\right.
$$

with $f(t)=F\left(u_{t}\right)$. In turn, given $f \in L^{1}(0, T ; X)$, a continuous function $u:[0, T] \rightarrow$ $X$ with $u(0)=\varphi(0)$ is a strong solution to $(\mathrm{CP})$ if it is locally absolutely continuous and differentiable a.e. $t \in[0, T]$ and fulfills $(\mathrm{CP})$ pointwise a.e. $t \in[0, T]$. It is a mild solution to $(\mathrm{CP})$ if, given any $\epsilon>0$, there exists an $\epsilon$-discrete-scheme approximation $D_{B}\left(t_{0}, \ldots, t_{N} ; u_{0}, \ldots, u_{N} ; f_{1}, \ldots f_{n}\right)$ consisting of an $\epsilon$-partition of the interval $[0, T]$,

$$
0 \leq t_{0}<t_{1}<\ldots<t_{N} \leq T, t_{0}<\epsilon, T-t_{N}<\epsilon, t_{i}-t_{i-1}<\epsilon, i \in\{1, \ldots, N\}
$$

and elements $\left\{u_{0}, u_{1}, \ldots, u_{N}\right\}$, and $\left\{f_{1}, f_{2}, \ldots, f_{N}\right\}$ in $X$ such that

$$
\begin{aligned}
& u_{i} \in D(B), i \in\{1, \ldots, N\}, \\
& \frac{u_{i}-u_{i-1}}{t_{i}-t_{i-1}}+B u_{i} \ni f_{i}, i \in\{1, \ldots, N\}, \\
& \sum_{1}^{N} \int_{t_{i-1}}^{t_{i}}\left\|f_{i}-f(\tau)\right\| d \tau<\epsilon, \quad \text { and }
\end{aligned}
$$


such that, if the step function $u_{\epsilon}:\left[t_{0}, t_{N}\right] \rightarrow X$ is defined by

$$
u_{\epsilon}(t)= \begin{cases}u_{0} & t=t_{0}, \\ u_{i} & t \in\left(t_{i-1}, t_{i}\right], i \in\{1, \ldots, N\},\end{cases}
$$

then $\left\|u_{\epsilon}(t)-u(t)\right\|<\epsilon$ uniformly over $t \in\left[t_{0}, t_{N}\right]$.

Recall that strong solutions are mild solutions, and that the converse requires special circumstances (which, in the context of (PFDE), will be considered in section $4)$.

For all these notions and the general theory of accretive sets and evolution equations, the reader is referred to [7, 48].

\section{Main Results on EXISTEnCE AND FLOW INVARIANCE}

In this section, we formulate and discuss our results on existence and flow invariance of mild solutions to (PFDE). The proofs will be given in section 3 below.

2.A. The initial-history space. Given a Banach (state) space $X$, and letting $I=(-\infty, 0]$ (infinite delay), or $I=[-R, 0]$ for some $R>0$ (finite delay), the initialhistory space is assumed to be a Banach space $(E,\|\cdot\|)$ of continuous functions $\varphi: I \rightarrow X$ with the following properties:

(E1) (a) For all $\varphi \in E,\|\varphi(0)\| \leq\|\varphi\|$.

(b) For all $x \in X, \tilde{x} \in E$, where $\tilde{x}(s) \equiv x, s \in I$, and

(c) there exists $C_{E} \geq 1$ such that $\|\tilde{x}\| \leq C_{E}\|x\|$ for all $x \in X$.

(d) For $\varphi,\left(\varphi_{n}\right)_{n}$ in $E$, if $\left\|\varphi_{n}-\varphi\right\| \rightarrow 0$, then (1) $\left\|\varphi_{n}(s)-\varphi(s)\right\| \rightarrow 0$ for all $s \in I$, and $(2) \int_{\alpha}^{\beta} \varphi_{n}(s) d s \rightarrow \int_{\alpha}^{\beta} \varphi(s) d s$ for all $\alpha, \beta \in I, \alpha<\beta$.

(E2) If $\lambda>0, x \in X, \psi \in E$, and $\varphi_{\lambda, x}^{\psi} \in C^{1}(I ; X)$ is the solution to $\varphi-\lambda \varphi^{\prime}=\psi$ with $\varphi(0)=x$, then $\varphi_{\lambda, x}^{\psi} \in E$, and $\left\|\varphi_{\lambda, x}^{\psi}\right\| \leq \max \{\|x\|,\|\psi\|\}$.

(E3) (a) If $x: I \cup[0, \infty) \rightarrow X$ is continuous, and $x_{\mid I} \in E$, then

(i) $x_{t} \in E$ for all $t \geq 0$, and

(ii) the map $\left\{t \rightarrow x_{t}\right\}$ is continuous from $\mathbb{R}^{+}$into $E$.

(b) There exist $M_{0} \geq 1$, and a locally bounded function $M_{1}: \mathbb{R}^{+} \rightarrow \mathbb{R}^{+}$ such that, given $x: I \cup[0, \infty) \rightarrow X$ as in (a) above, $\left\|x_{t}\right\| \leq M_{0}\left\|x_{0}\right\|+M_{1}(t) \max _{0 \leq s \leq t}\|x(s)\|$ for all $t \geq 0$.

(Concerning these axioms for $E$, compare [28, 30.)

Examples. In the finite-delay case, usually, the initial-history space will be $E=$ $C([-R, 0] ; X)$ with sup-norm. For the infinite-delay case $I=(-\infty, 0]$, the most prominent initial-history spaces are weighted sup-norm spaces of the type $E_{v}=$ $\left\{\varphi \in C\left(\mathbb{R}^{-}, X\right): v \varphi \in B U C\left(\mathbb{R}^{-} ; X\right)\right\}$, with norm $\|\varphi\|_{v}:=\sup \{v(s)\|\varphi(s)\| \mid s \in$ $\left.\mathbb{R}^{-}\right\}$, where the (weight-) function $v: \mathbb{R}^{-} \rightarrow(0,1]$ has the following properties:

(v1) $v$ is continuous, nondecreasing, and $v(0)=1$;

(v2) $\lim _{u \rightarrow 0^{-}} v(s+u) / v(s)=1$ uniformly over $s \in \mathbb{R}^{-}$.

Typical such weight functions are $v(s) \equiv 1$ (with, in this case, $E_{v}=B U C\left(\mathbb{R}^{-}, X\right)$ with sup-norm), $v(s)=e^{\mu s}$, or $v(s)=(1+|s|)^{-\mu}, \mu>0$ (spaces of "fading memory type"). (The Banach spaces $E_{v}$ are sometimes called $U C_{g}$-spaces, $v=1 / g$, and have been considered by various authors, cf. 30.)

Aside from $E_{v}$, also the following subspaces fulfill axioms (E1)-(E3):

(a) $E_{v_{l}}=\left\{\varphi \in E_{v} \mid \lim _{s \rightarrow-\infty} v(s) \varphi(s)\right.$ exists $\}$, and

(b) $E_{v_{0}}=\left\{\varphi \in E_{v} \mid \lim _{s \rightarrow-\infty} v(s) \varphi(s)=0\right\}$, in case $\lim _{s \rightarrow-\infty} v(s)=0$. 
2.B. The framework for (PFDE). Given an initial-history space $E$ as in 2.A, we start from the following assumptions:

(A1) $B \subset X \times X$ is $\omega$-accretive for some $\omega \in \mathbb{R}$.

(A2) $\hat{X} \subset X$ is a closed subset of $X$ with $\hat{X} \cap D(B) \neq \emptyset$.

(A3) (a) $\hat{E}_{0}=\{\varphi \in E \mid \varphi(0) \in \operatorname{cl}(D(B) \cap \hat{X})\}$.

(b) $\hat{E} \subset \hat{E}_{0}$ is a closed subset of $\hat{E}_{0}$.

(c) If $x \in \hat{X} \cap D(B), \psi \in \hat{E}$, and $\lambda>0$ is sufficiently small, then $\varphi_{\lambda, x}^{\psi} \in \hat{E}$, where $\varphi_{\lambda, x}^{\psi}$ is the solution $\varphi \in E$ to $\varphi-\lambda \varphi^{\prime}=\psi, \varphi(0)=x$.

(A4) The operator $F: \hat{E}_{0} \rightarrow X$ is such that

(a) $F: \hat{E}_{0} \rightarrow X$ is continuous;

(b) there exists $L_{F}>0$ such that, if $\varphi_{1}, \varphi_{2} \in \hat{E}_{0}$ with $\varphi_{1}(0)=\varphi_{2}(0)$, then $\left\|F \varphi_{1}-F \varphi_{2}\right\| \leq L_{F}\left\|\varphi_{1}-\varphi_{2}\right\|$, and

(c) there exists $M_{F}>0$ such that, if $\varphi_{1}, \varphi_{2} \in \hat{E}_{0}$ with $\left\|\varphi_{1}-\varphi_{2}\right\|=$ $\left\|\varphi_{1}(0)-\varphi_{2}(0)\right\|$, then $\left[\varphi_{1}(0)-\varphi_{2}(0), F \varphi_{1}-F \varphi_{2}\right] \leq M_{F}\left\|\varphi_{1}-\varphi_{2}\right\|$.

We are now in a position to formulate the main result of this paper.

Theorem 2.1. Given the assumptions (A1)-(A4), assume that

$$
\liminf _{\lambda \rightarrow 0^{+}} \frac{1}{\lambda} \operatorname{dist}(\psi(0)+\lambda F(\psi) ;(I+\lambda B)(D(B) \cap \hat{X}))=0 \quad \text { for all } \quad \psi \in \hat{E} .
$$

Then we have:

(a) For all $\psi \in \hat{E}$ there exists a global mild solution $u_{\psi}$ to

$$
\text { (PFDE) }\left\{\begin{array}{l}
\dot{u}(t)+B u(t) \ni F\left(u_{t}\right), t \geq 0, \\
u_{0}=\psi
\end{array}\right.
$$

with $\left(u_{\psi}\right)_{t} \in \hat{E}$ for all $t \geq 0$. In particular, $u_{\psi}(t) \in \hat{X}$ for all $t \geq 0$.

(b) If, in addition, either $\left.F\right|_{\hat{E}}: \hat{E} \rightarrow X$ is locally Lipschitz-continuous on $\hat{E}$, or there exists $M_{0}>0$ such that $[\varphi(0)-\psi(0), F \varphi-F \psi] \leq M_{0}\|\varphi-\psi\|$ for all $\varphi, \psi \in \hat{E}$, then, for any $\psi \in \hat{E}$, the mild solution $u_{\psi}$ as in (a) is unique amongst all mild solutions $u$ to (PFDE) with the property that $u_{t} \in \hat{E}$ for all $t \geq 0$.

(c) If $\psi_{1}, \psi_{2} \in \hat{E}$, then, for the corresponding mild solutions $u_{\psi_{1}}$ and $u_{\psi_{2}}$ as in (a),

$$
\left\|\left(u_{\psi_{1}}\right)_{t}-\left(u_{\psi_{2}}\right)_{t}\right\| \leq e^{\gamma t}\left\|\psi_{1}-\psi_{2}\right\|
$$

for all $t \geq 0$, where $\gamma=\max \left\{0, \omega+M_{F}\right\}$, and

$$
\begin{aligned}
& e^{-\omega t}\left\|u_{\psi_{1}}(t)-u_{\psi_{2}}(t)\right\|-e^{-\omega s}\left\|u_{\psi_{1}}(s)-u_{\psi_{2}}(s)\right\| \\
& \leq \int_{s}^{t} e^{-\omega \tau}\left[u_{\psi_{1}}(\tau)-u_{\psi_{2}}(\tau), F\left(\left(u_{\psi_{1}}\right)_{\tau}\right)-F\left(\left(u_{\psi_{2}}\right)_{\tau}\right)\right] d \tau \\
& \leq \int_{s}^{t} e^{-\omega \tau}\left\|F\left(\left(u_{\psi_{1}}\right)_{\tau}\right)-F\left(\left(u_{\psi_{2}}\right)_{\tau}\right)\right\| d \tau
\end{aligned}
$$

for all $0 \leq s \leq t$.

Remarks 2.2. 1. If, in addition to the assumptions (A1) and (A2),

$$
(c l D(B)) \cap \hat{X} \subset R(I+\lambda B) \text { and } J_{\lambda}{ }^{B}((c l D(B)) \cap \hat{X}) \subset \hat{X}
$$

for all $\lambda>0$ small enough, then $\operatorname{cl}(D(B) \cap \hat{X})=(\operatorname{cl} D(B)) \cap \hat{X}$, and thus, in Theorem 2.1, the set $\hat{E}_{0}$ can be chosen as $\hat{E}_{0}=\{\varphi \in E \mid \varphi(0) \in(\operatorname{cl} D(B)) \cap \hat{X}\}$. Notice that, with regard to solvability of (PFDE), the set $(\operatorname{cl} D(B)) \cap \hat{X}$ is the 
largest possible set of initial values: as we strive for flow-invariance of $\hat{X}$, we need $u(0)=\psi(0) \in \hat{X}$, and as we need existence of mild solutions, we need $u(0)=$ $\psi(0) \in \operatorname{cl} D(B)$.

2. In proposition (b) of Theorem 2.1, local Lipschitz-continuity of $F$ on $\hat{E}$ means that, given any $\psi \in \hat{E}$, there exist $\delta>0$ and $M>0$ such that $\left\|F \psi_{1}-F \psi_{2}\right\| \leq$ $M\left\|\psi_{1}-\psi_{2}\right\|$ for all $\psi_{1}, \psi_{2} \in \hat{E}$ with $\left\|\psi_{i}-\psi\right\|<\delta, i \in\{1,2\}$.

We now turn to various consequences of Theorem 2.1.

First, we note that, if $\hat{E}=\hat{E}_{0}$, then (A3) (c) is automatically fulfilled. This leads to the following consequence of Theorem 2.1 .

Theorem 2.3. Given the assumptions (A1), (A2), (A3) (a), and (A4), assume that

$(\mathrm{STC})_{0} \quad \liminf _{\lambda \rightarrow 0^{+}} \frac{1}{\lambda} \operatorname{dist}(\psi(0)+\lambda F(\psi) ;(I+\lambda B)(D(B) \cap \hat{X}))=0 \quad$ for all $\psi \in \hat{E}_{0}$.

Then we have:

For all $\psi \in \hat{E}_{0}$ there exists a global mild solution $u_{\psi}$ to

$$
(\text { PFDE })\left\{\begin{array}{l}
\dot{u}(t)+B u(t) \ni F\left(u_{t}\right), t \geq 0, \\
u_{0}=\psi
\end{array}\right.
$$

with $u_{\psi}(t) \in \hat{X}$ for all $t \geq 0$.

Moreover, propositions (b) and (c) of Theorem 2.1 hold with $\hat{E}$ being replaced by $\hat{E}_{0}$.

We note in passing, however, that, for concrete problems, validity of the subtangential condition for all $\psi \in \hat{E}_{0}$ rather than just for all $\psi$ in a suitably chosen closed subset $\hat{E}$ of $\hat{E}_{0}$ may be hard to come by. This will become clear in our applications to concrete models; see section 5 .

In the results for the ordinary delay case of $B=0$, the set $\hat{E}$ has been taken as $\hat{E}=\left\{\varphi \in \hat{E}_{0} \mid \varphi(s) \in \hat{X}\right.$ for all $\left.s \in I\right\}$ with $\hat{X} \subset X$ closed and convex. We take up this special case of Theorem 2.1, and note that in this case, too, (A3) (c) is automatically fulfilled (as $\hat{X}$ is (closed and) convex).

Theorem 2.4. Given the assumptions (A1), (A2), (A3) (a), and (A4), assume in addition that $\hat{X}$ is convex, and let $\hat{E}=\left\{\varphi \in \hat{E}_{0} \mid \varphi(s) \in \hat{X}\right.$ for all $\left.s \in I\right\}$. Then, if

$$
\liminf _{\lambda \rightarrow 0^{+}} \frac{1}{\lambda} \operatorname{dist}(\psi(0)+\lambda F(\psi) ;(I+\lambda B)(D(B) \cap \hat{X}))=0 \quad \text { for all } \psi \in \hat{E},
$$

all of propositions (a)-(c) of Theorem 2.1 hold.

We now turn to a specialization of Theorem 2.1 of a different kind. Notice that the subtangential condition (STC) of Theorem 2.1 requires approximations by elements in the range of $(I+\lambda B)$. Obviously, it would be much more convenient for concrete applications if the operator $B$ could be eliminated from condition (STC). Taking up an idea by [9, Lemma 4.2] (for the special case of no delay), there is one significant situation under which this can be achieved, namely, if the resolvents of $B$ have $\hat{X}$ contained in their domain and leave $\hat{X}$ invariant. The precise statement is as follows. 
Theorem 2.5. Given the assumptions (A1)-(A4), assume, in addition, that

$$
R(I+\lambda B) \supset \hat{X} \quad \text { and } \quad J_{\lambda}{ }^{B} \hat{X} \subset \hat{X} \quad \text { for all }>0 \quad \text { small enough. }
$$

Then, if

$$
(\mathrm{STC})_{X} \quad \liminf _{\lambda \rightarrow 0^{+}} \frac{1}{\lambda} \operatorname{dist}(\psi(0)+\lambda F(\psi) ; \hat{X})=0 \quad \text { for all } \quad \psi \in \hat{E},
$$

all of propositions (a)-(c) of Theorem 2.1 hold.

Thus, the flow-invariance problem is reduced to two independent conditions:, one on $\hat{X}, \hat{E}$ and $F$, and one on the resolvents of $B$. In particular, if the subtangential condition $(\mathrm{STC})_{X}$ is fulfilled, then flow-invariance of $\hat{X}$ and $\hat{E}$ hold for all $\omega-\mathrm{m}$ accretive operators, the resolvents of which leave $\hat{X}$ invariant.

Actually, there is a prominent subclass of accretive operators for which this reduction of (STC) to (STC) $X$ can be achieved for natural choices of $\hat{X}$. Recall (cf. [6]) that, for $\Omega$ a $\sigma$-finite measure space, an operator $B \subset L^{1}(\Omega) \times L^{1}(\Omega)$ is said to be $m$-completely accretive if (a) it is m-accretive, and if (b) its resolvents are both order-preserving and contractive in all $L^{p}$-norms for $1 \leq p \leq \infty$. Thus, if for a state-space $X=L^{1}(\Omega), \hat{X}$ is chosen as an order interval of the form $\hat{X}=$ $[0, \beta]:=\left\{x \in L^{1}(\Omega) \mid 0 \leq x(\omega) \leq \beta\right.$ a.e. $\left.\omega \in \Omega\right\}$ with $\beta>0$, then (2.4) is automatically fulfilled for any m-completely accretive operator $B \subset L^{1}(\Omega) \times L^{1}(\Omega)$ with $(0,0) \in B$.

We state this special case of Theorem 2.5 on invariance of order-intervals separately. It will turn out to be particularly useful for concrete models from population dynamics; see section 5 .

Corollary 2.6. Given a $\sigma$-finite measure space $(\Omega ; \Sigma ; \mu)$, let $X=L^{1}(\Omega)$, and, for $\beta>0, \hat{X}=[0, \beta]:=\left\{x \in L^{1}(\Omega) \mid 0 \leq x(\omega) \leq \beta\right.$ a.e. $\left.\omega \in \Omega\right\}$, and let $B \subset L^{1}(\Omega) \times L^{1}(\Omega)$ be an m-completely accretive operator with $(0,0) \in B$. Then, if assumptions (A3) and (A4) hold, and if

$$
(\mathrm{STC})_{X} \quad \liminf _{\lambda \rightarrow 0^{+}} \frac{1}{\lambda} \operatorname{dist}(\psi(0)+\lambda F(\psi) ; \hat{X})=0 \quad \text { for all } \psi \in \hat{E},
$$

all of propositions (a)-(c) of Theorem 2.1 hold.

In closing this section, we note the relationship between the subtangential condition (STC) of Theorem 2.1 and the local range condition that was used in 67, 70] for existence and flow-invariance ([67, section 2, (A2)]): For $\psi \in \hat{E}$, and $\lambda>0$ with $\lambda \gamma<1$,

$$
(\mathrm{LRC}) \quad\left(\psi(0)+\lambda F\left(\varphi_{\lambda, x}^{\psi}\right)\right) \in(I+\lambda B)(D(B) \cap \hat{X}) \quad \text { for all } x \in \hat{X} .
$$

Lemma 2.7. Under conditions (A1)-(A4), assume that $\left(\psi(0)+\lambda F\left(\varphi_{\lambda, \psi(0)}^{\psi}\right)\right) \in$ $(I+\lambda B)(D(B) \cap \hat{X}) \quad$ for all $\psi \in \hat{E}$, and all $\lambda>0$ small enough (which is implied by (LRC)). Then (STC) of Theorem 2.1 holds.

(Otherwise, 67, Thm. 2.1] and Theorem 2.1 above are incomparable with regard to the assumptions on $\hat{E}$ and $F$.) 
Remarks 2.8. Relations to existing results. 1. The global case: If $B \subset X \times X$ is $\omega$-m-accretive, and $F: E \rightarrow X$ is globally Lipschitz, then, with $\hat{X}:=X, \hat{E}_{0}=$ $\{\varphi \in E \mid \varphi(0) \in \operatorname{cl} D(B)\}$, and $\hat{E}:=\hat{E}_{0}$, all of (A1)-(A4) and (STC) (as well as (2.4) and (2.5)) hold trivially. Thus, both of Theorems 2.1 and 2.5 contain the existence results for this global case; cf. [11, 12, 25, 28, 31, 32, 37, 38, 50, Chapters B-IV.3, C-IV.3], [58, 59, 76, 77, 79, 80, 81, 82, as well as [14, 65, 87] for further references. (In one or the other of the above references, there is existence of strong solutions for the case of $X$ reflexive and $\varphi \in \hat{D}(A)$ - the generalized domain ([15]) of the operator $A$ defined by (3.1) below. For $B \omega-$ m-accretive, this follows (from Theorem 2.1 and) from general regularity properties of mild solutions even for the more general case of $X$ having the Radon-Nikodym property; compare [7, Cor. 7.5].)

A further special case of Theorems 2.1 and 2.5 is the case of $B: D(B) \subset X \rightarrow$ $X$ single-valued linear and a Hille-Yosida operator, i.e., fulfilling the Hille-Yosida conditions, but not necessarily being densely defined. To me, it seems largely being overlooked that such operators are $\omega-$ m-accretive for an equivalent norm on $X$ and thus fall under the general (even nonlinear) context. In particular, the existence results for (PFDE) with $B$ Hille-Yosida, and $F$ globally Lipschitz are special cases of both Theorems 2.1 and 2.5, and of [67, Thm. 2.1]; for references, cf. [1, 23].

We note in passing that, for $B \omega-\mathrm{m}$-accretive, local existence results for (PFDE) can as well be achieved for $F$ (globally defined and) Lipschitz on bounded sets by simply considering suitable truncations of $F$ (being globally Lipschitz).

2. Subtangential conditions: (a) For the case of the ordinary differential delay equation $\dot{u}(t)=F\left(u_{t}\right)$, i.e. $B \equiv 0$, condition (STC) reduces to the corresponding subtangential conditions of [39, 40, 75] (condition $(\mathrm{STC})_{X}$ of $(2.5)$ above), while 41 uses a related, but different condition. (Also, the results, partly are different with respect to the assumptions on $F$, and allow $F$ to be $t$-dependent.)

(b) Specialized to the non-delay case of the evolution equation $\dot{u}(t)+B u(t) \ni f(u(t))$, with $B \omega-$ m-accretive, the subtangential condition $\liminf _{\lambda \rightarrow 0^{+}} \frac{1}{\lambda} \operatorname{dist}(x+\lambda f(x) ;(I+\lambda B)(D(B) \cap \hat{X}))=0 \quad$ for all $\quad x \in \operatorname{cl} D(B) \cap \hat{X}$ is a sufficient condition for flow-invariance of $\hat{X}$ under various assumptions on $f$; cf. 9]. For this special case, the references [2, 9, 10, 42, Ch. VIII], and [54, 60] (and many others, compare the references in [9]) also use a weaker subtangential condition which, in particular cases, is also necessary for flow-invariance. Given in terms of the semigroup generated by $-B$, though, it is not as useful as a sufficient condition, for, what is known in concrete instances is the operator $B$, but not the semigroup it generates. More to the point, in contrast to these references, beyond (STC), we have no further 'generating' assumption on $B$, much less $\omega-\mathrm{m}-$ accretivity, or even a range condition.

(c) For the semilinear case of $B$ linear, and $-B$ generating a $C_{0}$-semigroup of bounded linear operators on $X$, versions of Theorem 2.1 (even for $F t$-dependent) are given in 43, 44, 55, 56 under subtangential conditions involving the semigroup generated by $-B$. (While, in [43, 44, and 56, Ch. 5.3], these are weaker than (STC), the one in [55] is somewhat incomparable, as it includes initial histories with values in a neighbourhood of $\hat{X}$, which would thus not work for our models such as (1.1), or those in section 5.)

3. The nonautonomous case: We would want to mention that, for the nonautonomous global case, with $F(t, \cdot)$ globally Lipschitz, and $B(t, \cdot)$ m-accretive, there 
is a well-established solution theory as well. Here, we only mention a few representative papers [19, 20, 21, 24, 33, 34, 35, 51, 62, 66], and refer to 65] for a rather complete list of references. Flow-invariance results for the fully nonautonomous case require additional techniques and will be treated elsewhere.

\section{Proofs of Theorems 2.1 and 2.5}

Our methods of proof will entirely be based on the technique of transforming the (original) problem (PFDE) in (the state space) $X$ into a Cauchy problem in (the initial-history space) $E$. We start with a brief outline of the program of this technique of proof.

We associate with (PFDE) the operator $A$ in $E$ defined by

$$
\left\{\begin{array}{l}
D(A)=\left\{\varphi \in \hat{E}_{0} \mid \varphi^{\prime} \in E, \varphi(0) \in D(B), \varphi^{\prime}(0) \in F \varphi-B \varphi(0)\right\} \\
A \varphi:=-\varphi^{\prime}, \varphi \in D(A) .
\end{array}\right.
$$

Given the assumptions of Theorem 2.1, we shall prove the following assertions:

(S1): The operator $A$ is $\gamma$-accretive in $E$ with $\gamma=\max \left\{0, \omega+M_{F}\right\}$.

(S2): For every $\varphi \in(\hat{E} \cap c l D(A))$, there exists a unique mild solution $\phi \varphi: \mathbb{R}^{+} \rightarrow E$ to the Cauchy problem in $E$ corresponding to $A$,

$$
(\mathrm{CP})\left\{\begin{array}{l}
\dot{\phi}(t)+A \phi(t)=0, \quad t \geq 0 \\
\phi(0)=\varphi
\end{array}\right.
$$

The solution semigroup $(S(t))_{t \geq 0}$ generated by $-A$ via $S(t) \varphi:=\phi \varphi(t)$ leaves $(\hat{E} \cap$ cl $D(A))$ invariant.

(S3): $\operatorname{cl} D(A)=\hat{E}_{0}($ and thus, $(\hat{E} \cap \operatorname{cl} D(A))=\hat{E})$.

(S4): If $\varphi \in \hat{E}$, and $u_{\varphi}: I \cup \mathbb{R}^{+} \rightarrow X$ is defined by

$$
u_{\varphi}(t)= \begin{cases}\varphi(t) & t \in I \\ (S(t) \varphi)(0) & t \geq 0\end{cases}
$$

then $S(t) \varphi=\left(u_{\varphi}\right)_{t}$ for $t \geq 0$ (i.e., $(S(t))_{t \geq 0}$ acts as a translation).

(S5) For $\varphi \in \hat{E}$, the function $u_{\varphi}$ from (S4) is a global mild solution to (PFDE).

Obviously, this series of assertions will serve to prove assertions (a) and (c) (2.1) of Theorem 2.1.

This approach to solving (PFDE) via the Cauchy problem in $E$ as in (S2) is quite common and actually goes back to the very beginning of treating this problem; cf. [11, 12, 19, 20, 51, 58, 76, 77, 79, 80. (For a detailed list in this respect, compare 65] and 67] and the references therein.) Although (S1) is in a more general setup than in previous papers, its proof is somewhat routine, and assertion (S4) is due to 58 .

The real heart of the matter is assertion (S2). With regard to the flow-invariance result for the Cauchy problem as reported in Remarks 2.8, 2 (b), above, a direct way of establishing (S2) would be to translate the subtangential condition (STC) of Theorem 2.1 in $X$ into the subtangential condition for flow-invariance of $\hat{E}$ for the Cauchy problem in $E$, i.e.,

$$
(\mathrm{STC})_{A} \quad \liminf _{\lambda \rightarrow 0^{+}} \frac{1}{\lambda} \operatorname{dist}(\psi ;(I+\lambda A)(D(A) \cap \hat{E}))=0
$$

for all $\psi \in \hat{E}$. However, to me, this particular approximation of $\psi \in \hat{E}$ by $\left(\varphi-\lambda \varphi^{\prime}\right)$ with $\varphi$ simultaneously in $D(A)$ and in $\hat{E}$, does not seem to be possible under assumption (STC). As can be read from the technique of the subsequent proof 
of assertion (S2), for the special case of $\hat{E}=\left\{\varphi \in \hat{E}_{0} \mid \varphi(s) \in \hat{X}, s \in I\right\}$, it would be possible, though, if only the subtangential condition (STC) of Theorem 2.1 would be assumed to hold (not only for $\varphi \in \hat{E}$, but even) for all $\varphi \in \hat{E}_{0}$ such that $\operatorname{dist}(\varphi(s) ; \hat{X}) \leq \rho$ for all $s \in I$, and some $\rho>0$. For a flow-invariance subtangential condition of this 'extended' type, see [55] (for this special case of $\hat{E}$, and, moreover, for $B$ linear (single-valued) and the generator of a $C_{0}$-semigroup). However, for our concrete population models in the state space $L^{1}$, the operator $F$ may not even be defined on such $\gamma$-neighbourhoods of $\hat{E}$; compare the examples in section 5. This is why we have to base our proof of assertion (S2) on a "separate subtangential condition" by [57]; see below.

3.1. Proof of Theorem 2.1. We proceed by proving assertions (S1)-(S5).

Step 1: Proof of (S1). Let $\gamma=\max \left\{0, \omega+M_{F}\right\}$, and let $\lambda>0$ such that $\lambda \gamma<1$. We have to show that

$$
\left\|\varphi_{1}-\varphi_{2}\right\| \leq(1-\lambda \gamma)^{-1}\left\|\left(\varphi_{1}-\lambda \varphi_{1}^{\prime}\right)-\left(\varphi_{2}-\lambda \varphi_{2}^{\prime}\right)\right\|
$$

for all $\varphi_{1}, \varphi_{2} \in D(A)$. To this end, let $\varphi=\varphi_{1}-\varphi_{2}$, and $\psi_{i, \lambda}=\varphi_{i}-\lambda \varphi_{i}^{\prime}$. Then we have $\varphi-\lambda \varphi^{\prime}=\psi_{1, \lambda}-\psi_{2, \lambda}, \varphi(0)=\varphi_{1}(0)-\varphi_{2}(0)$, so that, according to (E2), $\left\|\varphi_{1}-\varphi_{2}\right\|=\|\varphi\| \leq \max \left\{\left\|\varphi_{1}(0)-\varphi_{2}(0)\right\|,\left\|\psi_{1, \lambda}-\psi_{2, \lambda}\right\|\right\}$. In case the second term is the maximum, we are done. Otherwise, using (a) of (E1),

$$
\begin{aligned}
& \left\|\varphi_{1}-\varphi_{2}\right\|=\left\|\varphi_{1}(0)-\varphi_{2}(0)\right\|, \quad \text { and thus, by }(\mathrm{c}) \text { of }(\mathbf{A} 4), \\
& \left\langle x^{*}, F \varphi_{1}-F \varphi_{2}\right\rangle \leq M_{F}\left\|\varphi_{1}-\varphi_{2}\right\| \text { for all } x^{*} \in J\left(\varphi_{1}(0)-\varphi_{2}(0)\right) .
\end{aligned}
$$

Moreover, since $\varphi_{i} \in D(A), \varphi_{i}(0)=J_{\lambda}{ }^{B}\left(\psi_{i, \lambda}(0)+\lambda F\left(\varphi_{i}\right)\right), i \in\{1,2\}$. This implies that

$$
\frac{1}{\lambda}\left(\psi_{i, \lambda}(0)+\lambda F\left(\varphi_{i}\right)-\varphi_{i}(0)\right) \in B \varphi_{i}(0), i \in\{1,2\} .
$$

Thus, as $B$ is $\omega$-accretive, there exists $x^{*} \in J\left(\varphi_{1}(0)-\varphi_{2}(0)\right)$ such that, using (3.4), (3.5) and (3.6),

$$
\begin{aligned}
-\lambda \omega\left\|\varphi_{1}(0)-\varphi_{2}(0)\right\| \leq & \left\langle x^{*}, \psi_{1, \lambda}(0)+\lambda F\left(\varphi_{1}\right)-\left(\psi_{2, \lambda}(0)+\lambda F\left(\varphi_{2}\right)\right)\right. \\
& \left.-\left(\varphi_{1}(0)-\varphi_{2}(0)\right)\right\rangle \\
= & \left\langle x^{*}, \psi_{1, \lambda}(0)-\psi_{2, \lambda}(0)\right\rangle+\lambda\left\langle x^{*}, F\left(\varphi_{1}\right)-F\left(\varphi_{2}\right)\right\rangle \\
& -\left\|\varphi_{1}(0)-\varphi_{2}(0)\right\| \\
\leq & \left\|\psi_{1, \lambda}-\psi_{2, \lambda}\right\|+\left(\lambda M_{F}-1\right)\left\|\varphi_{1}-\varphi_{2}\right\| .
\end{aligned}
$$

According to (3.4), rearranging yields $\left(1-\lambda\left(\omega+M_{F}\right)\right)\left\|\varphi_{1}-\varphi_{2}\right\| \leq\left\|\psi_{1, \lambda}-\psi_{2, \lambda}\right\|$, so that, by the definition of $\gamma$, we are done in any event.

Step 2: Proof of (S2). As indicated in the introductory remarks to this section, it does not seem to be possible to derive from (STC) the usual subtangential condition $(\mathrm{STC})_{A}$ for flow-invarianve of $\hat{E}$. Instead, we shall base our proof on the following separate subtangential condition developed by M. Pierre:

Theorem ([57, Thm. 2]). Assume that $C \subset Z \times Z$ is an $\alpha$-accretive operator in a Banach space $Z$, and let $F$ be a closed subset of $Z$. Assume, moreover, that the pair $(C, F)$ fulfills the following condition:

$$
H(0,0) \quad\left\{\begin{array}{l}
\forall z \in F, \forall \epsilon>0, \exists \lambda \in(0, \epsilon], \exists\left(z_{\lambda}, w_{\lambda}\right) \in C, \exists u_{\lambda} \in F \text { such that } \\
\left\|z-\left(z_{\lambda}+\lambda w_{\lambda}\right)\right\| \leq \lambda \epsilon, \quad \text { and } \quad\left\|z_{\lambda}-u_{\lambda}\right\| \leq \lambda \epsilon .
\end{array}\right.
$$


Then, for every $z \in F \cap c l D(C)$, there exists a unique mild solution $u(z ; \cdot): \mathbb{R}^{+} \rightarrow F$ of the Cauchy problem

$$
\text { (CP) }\left\{\begin{array}{l}
\dot{u}(t)+C u(t) \ni 0, \quad t \geq 0 \\
u(0)=z
\end{array}\right.
$$

The semigroup $(S(t))_{t \geq 0}$ defined by $S(t) z=u(z ; t), z \in F \cap c l D(C)$, is a semigroup of $\alpha$-contractions, i.e., $\left\|S(t) z_{1}-S(t) z_{2}\right\| \leq e^{\alpha t}\left\|z_{1}-z_{2}\right\|, z_{1}, z_{2} \in F \cap c l D(C), t \geq$ 0 , and leaves $F \cap c l D(C)$ invariant.

(Actually, in [57, Thm. 2], the result is given for the accretive case $\alpha=0$, but the method of proof works just as well for the general case.)

In the light of this result, in order to prove (S2), it is enough to show that the pair $(A, \hat{E})$ fulfills condition $H(0,0)$. This will be done by a series of steps.

Let $\psi \in \hat{E}$. Then, according to (STC),

$$
\left\{\begin{array}{l}
\exists \epsilon_{n} \rightarrow 0^{+}, \lambda_{n} \rightarrow 0^{+},\left(x_{n}, y_{n}\right) \in B, x_{n} \in \hat{X} \quad \text { such that } \\
\left(\lambda_{n}\right)^{-1}\left\|\psi(0)+\lambda_{n} F(\psi)-\left(x_{n}+\lambda_{n} y_{n}\right)\right\| \leq \epsilon_{n}
\end{array}\right.
$$

for all $n \in \mathbb{N}$.

Step 2.1: Claim: With $\varphi_{\lambda_{n}, x_{n}}^{\psi} \in \hat{E}$ as in (A3), (c),

$$
\left(\lambda_{n}\right)^{-1}\left\|\psi(0)+\lambda_{n} F\left(\varphi_{\lambda_{n}, x_{n}}^{\psi}\right)-\left(x_{n}+\lambda_{n} y_{n}\right)\right\| \rightarrow 0 .
$$

Proof. First, we prove that $\left\|x_{n}-\psi(0)\right\| \rightarrow 0$. To this end, and for later reference (in the proof of (S3)), we recall the following elementary fact: If $C \subset Z \times Z$ is an $\omega$-accretive operator in a Banach space $Z$, then, for all $\lambda>0$, with $\lambda \omega<1$, for all $z \in R(I+\lambda C)$, and for all $\left(z_{0}, w_{0}\right) \in C$,

$$
\begin{aligned}
& \left\|z-J_{\lambda}{ }^{C} z\right\| \leq\left\|z-z_{0}\right\|+\left\|J_{\lambda}{ }^{C}\left(z_{0}+\lambda w_{0}\right)-J_{\lambda}{ }^{C} z\right\| \leq\left\|z-z_{0}\right\| \\
& \quad+(1-\lambda \omega)^{-1}\left\|z_{0}+\lambda w_{0}-z\right\| \leq\left(1+(1-\lambda \omega)^{-1}\right)\left\|z-z_{0}\right\|+\lambda(1-\lambda \omega)^{-1}\left\|w_{0}\right\| .
\end{aligned}
$$

Applying (3.9) to $X, B, z=x_{n}+\lambda_{n} y_{n}$, and $\left(x_{0}, y_{0}\right) \in B$ arbitrary,

$$
\begin{aligned}
& \left\|\psi(0)-x_{n}\right\| \leq\left\|\psi(0)-\left(x_{n}+\lambda_{n} y_{n}\right)\right\|+\left\|\left(x_{n}+\lambda_{n} y_{n}\right)-J_{\lambda_{n}}^{B}\left(x_{n}+\lambda_{n} y_{n}\right)\right\| \\
\leq & \left\|\psi(0)-\left(x_{n}+\lambda_{n} y_{n}\right)\right\|+\left(1+\left(1-\lambda_{n} \omega\right)^{-1}\right)\left\|x_{n}+\lambda_{n} y_{n}-x_{0}\right\| \\
& +\lambda_{n}\left(1-\lambda_{n} \omega\right)^{-1}\left\|y_{0}\right\| \\
\leq & \left\|\psi(0)-\left(x_{n}+\lambda_{n} y_{n}\right)\right\|+\left(1+\left(1-\lambda_{n} \omega\right)^{-1}\right)\left[\left\|x_{n}+\lambda_{n} y_{n}-\psi(0)\right\|\right. \\
& \left.+\left\|\psi(0)-x_{0}\right\|\right]+\lambda_{n}\left(1-\lambda_{n} \omega\right)^{-1}\left\|y_{0}\right\| .
\end{aligned}
$$

As, by (3.7), $\left\|\psi(0)-\left(x_{n}+\lambda_{n} y_{n}\right)\right\| \leq \lambda_{n} \epsilon_{n}+\lambda_{n}\|F(\psi)\| \rightarrow 0$, we thus have

$$
\limsup _{n \rightarrow \infty}\left\|\psi(0)-x_{n}\right\| \leq 2\left\|\psi(0)-x_{0}\right\| \quad \text { for all } x_{0} \in D(B) .
$$

As $\psi \in \hat{E}$, and thus $\psi(0) \in \operatorname{cl}(D(B) \cap \hat{X}) \subset \operatorname{cl} D(B)$, we thus conclude that $x_{n} \rightarrow \psi(0)$.

According to (E2), this implies that

$$
\left\|\varphi_{\lambda_{n}, x_{n}}^{\psi}-\varphi_{\lambda_{n}, \psi(0)}^{\psi}\right\| \rightarrow 0
$$

The next step towards the proof of (3.8) is to show that

$$
\left\|\psi-\varphi_{\lambda_{n}, \psi(0)}^{\psi}\right\| \rightarrow 0 .
$$


To this end, we consider the following auxiliary operator $C$ in $E$ :

$$
\left\{\begin{array}{l}
D(C)=\left\{\varphi \in E \mid \varphi^{\prime} \in E, \varphi^{\prime}(0)=0\right\} \\
C \varphi:=-\varphi^{\prime}, \varphi \in D(C)
\end{array}\right.
$$

As is easily checked, $C$ is a (linear) m-accretive operator with dense domain. (Actually, this fact can as well be read from 70, Thms. 2.1 and 2.6] for the special case of $B=0=F$, and $\hat{X}=X$, and $\hat{E}=E$.) Thus, $J_{\lambda}{ }^{C} \rho \rightarrow \rho$ as $\lambda \rightarrow 0^{+}$for all $\rho \in E$. However, $\varphi_{\lambda_{n}, \psi(0)}^{\psi}=J_{\lambda_{n}}^{C} \psi$, and so (3.11) is proved.

At this point, we combine (3.7), (3.10) and (3.11) to conclude that

$$
\begin{aligned}
& \left(\lambda_{n}\right)^{-1}\left\|\psi(0)+\lambda_{n} F\left(\varphi_{\lambda_{n}, x_{n}}^{\psi}\right)-\left(x_{n}+\lambda_{n} y_{n}\right)\right\| \\
& \leq\left(\lambda_{n}\right)^{-1}\left\|\psi(0)+\lambda_{n} F(\psi)-\left(x_{n}+\lambda_{n} y_{n}\right)\right\|+\left\|F\left(\varphi_{\lambda_{n}, x_{n}}^{\psi}\right)-F(\psi)\right\| \rightarrow 0,
\end{aligned}
$$

by continuity of $F$ on $\hat{E}$. This completes the proof of (3.8).

Step 2.2: As will become clear later on, the functions $\psi_{n}:=\varphi_{\lambda_{n}, x_{n}}^{\psi} \in \hat{E}$ turn out to serve for the approximating elements in $\hat{E}$ as required for the validity of $H(0,0)$ for the pair $(A, \hat{E})$. In order to determine the corresponding approximating elements in $D(A)$ (and for later reference in the proof of (S3)), we note the following auxiliary result.

Lemma 3.1. Given $\rho \in \hat{E}_{0}, x \in D(B) \cap \hat{X}, y \in B x$, and $\lambda>0$ such that $\lambda C_{E} L_{F}<$ 1 , let $\hat{E}_{0, x}:=\left\{\varphi \in \hat{E}_{0} \mid \varphi(0)=x\right\}$, and consider the following operator:

$$
\left\{\begin{array}{l}
T: \hat{E}_{0, x} \rightarrow \hat{E}_{0, x} \\
\varphi \mapsto\left\{s \mapsto e^{s / \lambda} x+\frac{1}{\lambda} e^{s / \lambda} \int_{s}^{0} e^{-\xi / \lambda}[\rho(\xi)+\{x+\lambda y-(\rho(0)+\lambda F(\varphi))\}] d \xi .\right.
\end{array}\right.
$$

Then $T$ has a fixed point $\hat{\phi} \in \hat{E}_{0, x}$, and $\hat{\phi} \in D(A)$.

Proof. First, note that, by (E1) (b) and (E2), the operator $T$ is well defined, and for $\varphi \in \hat{E}_{0, x}, T \varphi-\lambda(T \varphi)^{\prime}=\rho+x+\lambda y-(\rho(0)+\lambda F(\varphi))$, and $(T \varphi)(0)=x$. Thus, from (E2), (E1) (c), and (A4) (b),

$$
\left\|T \varphi_{1}-T \varphi_{2}\right\| \leq \lambda\left\|\widetilde{F \varphi_{1}}-\widetilde{F \varphi_{2}}\right\| \leq \lambda C_{E} L_{F}\left\|\varphi_{1}-\varphi_{2}\right\|
$$

for all $\varphi_{1}, \varphi_{2} \in \hat{E}_{0, x}$. According to our assumption on $\lambda$, $T$ has a fixed point $\hat{\varphi}$. Thus, $\hat{\varphi} \in \hat{E}_{0}, \hat{\varphi}(0)=x \in D(B)$, and $\hat{\varphi}-\lambda \hat{\varphi}^{\prime}=\rho+x+\lambda y-(\rho(0)+\lambda F(\hat{\varphi}))$, so that

$\hat{\varphi}^{\prime}(0)=\frac{1}{\lambda}[x-\rho(0)-(x+\lambda y)+\rho(0)+\lambda F(\hat{\varphi})]=F(\hat{\varphi})-y \in F(\hat{\varphi})-B x=F(\hat{\varphi})-B(\hat{\varphi}(0))$.

Thus, $\hat{\varphi} \in D(A)$ as well, and the lemma is proved.

Step 2.3: Applying Lemma 3.1 to $\rho=\psi,(x, y)=\left(x_{n}, y_{n}\right) \in B$, and $\lambda=\lambda_{n}$ as in (3.7), we get, for $n \in \mathbb{N}, \varphi_{n} \in D(A)$ with

$$
\varphi_{n}-\lambda_{n} \varphi_{n}^{\prime}=\psi+x_{n}+\lambda_{n} y_{n}-\left(\psi(0)+\lambda_{n} F\left(\varphi_{n}\right)\right), \quad \varphi_{n}(0)=x_{n} .
$$


Invoking the functions $\varphi_{\lambda_{n}, x_{n}}^{\psi}$ of Step 2.1 and applying (E2), in conjunction with (E1) (c) and (A4) (b), yield

$$
\begin{aligned}
& \left(\lambda_{n}\right)^{-1}\left\|\varphi_{\lambda_{n}, x_{n}}^{\psi}-\varphi_{n}\right\| \leq\left(\lambda_{n}\right)^{-1} C_{E}\left\|x_{n}+\lambda_{n} y_{n}-\left(\psi(0)+\lambda_{n} F\left(\varphi_{n}\right)\right)\right\| \\
& \quad \leq\left(\lambda_{n}\right)^{-1} C_{E}\left\|x_{n}+\lambda_{n} y_{n}-\left(\psi(0)+\lambda_{n} F\left(\varphi_{\lambda_{n}, x_{n}}^{\psi}\right)\right)\right\|+C_{E}\left\|F\left(\varphi_{\lambda_{n}, x_{n}}^{\psi}\right)-F\left(\varphi_{n}\right)\right\| \\
& \quad \leq\left(\lambda_{n}\right)^{-1} C_{E}\left\|x_{n}+\lambda_{n} y_{n}-\left(\psi(0)+\lambda_{n} F\left(\varphi_{\lambda_{n}, x_{n}}^{\psi}\right)\right)\right\|+C_{E} L_{F}\left\|\varphi_{\lambda_{n}, x_{n}}^{\psi}-\varphi_{n}\right\|
\end{aligned}
$$

for all $n \in \mathbb{N}$. Rearranging, and invoking (3.8), shows that

$$
\left(\lambda_{n}\right)^{-1}\left\|\varphi_{\lambda_{n}, x_{n}}^{\psi}-\varphi_{n}\right\| \rightarrow 0 \quad \text { as } \quad n \rightarrow \infty .
$$

Going back into the string of inequalities (3.13), this, in turn, implies that

$$
\left(\lambda_{n}\right)^{-1}\left\|x_{n}+\lambda_{n} y_{n}-\left(\psi(0)+\lambda_{n} F\left(\varphi_{n}\right)\right)\right\| \rightarrow 0 \quad \text { as } \quad n \rightarrow \infty .
$$

Thus, letting $\psi_{n}=\varphi_{\lambda_{n}, x_{n}}^{\psi}$, we have determined sequences $\left(\varphi_{n}\right)_{n} \in D(A),\left(\psi_{n}\right)_{n} \in$ $\hat{E}$, and $\lambda_{n} \rightarrow 0^{+}$such that, by (3.14), (3.15) and by (3.12) in conjunction with (E1) (c),

$$
\begin{aligned}
& \left(\lambda_{n}\right)^{-1}\left\|\psi_{n}-\varphi_{n}\right\| \rightarrow 0, \quad \text { and } \\
& \left(\lambda_{n}\right)^{-1}\left\|\psi-\left(\varphi_{n}-\lambda_{n} \varphi_{n}^{\prime}\right)\right\| \leq\left(\lambda_{n}\right)^{-1} C_{E}\left\|x_{n}+\lambda_{n} y_{n}-\left(\psi(0)+\lambda_{n} F\left(\varphi_{n}\right)\right)\right\| \rightarrow 0
\end{aligned}
$$

as $n \rightarrow \infty$. This serves to prove that condition $H(0,0)$ is fulfilled for the pair $(A, \hat{E})$, and completes the proof of (S2).

Step 3: Proof of (S3). We have to show that $D(A)$ is dense in $\hat{E}_{0}$. To this end, we shall need an auxiliary result, and we adopt the following notation in order to state it:

1. $E_{0}=\{\varphi \in E \mid \varphi(0)=0\}$.

2. For $\lambda>0$, the function $e_{\lambda}: I \rightarrow \mathbb{R}$ is defined by $e_{\lambda}(s)=\exp (s / \lambda), s \in I$.

Lemma 3.2. Under the assumptions $(\mathbf{E 1})-(\mathbf{E} 3)$, and (A1)-(A4), and with $\gamma=$ $\max \left\{0, \omega+M_{F}\right\}$, the following propositions hold:

(a) If $A_{0}$ is the operator in $E_{0}$ defined by

$$
D\left(A_{0}\right)=\left\{\varphi \in E_{0} \mid \varphi^{\prime} \in E_{0}\right\}, A_{0} \varphi=-\varphi^{\prime}, \varphi \in D\left(A_{0}\right),
$$

then $-A_{0}$ is the generator of the $C_{0}-$ semigroup $\left(T_{0}(t)\right)_{t \geq 0}$ of linear contractions $T_{0}(t): E_{0} \rightarrow E_{0}$ given by

$$
T_{0}(t) \varphi(s)= \begin{cases}0 & -t \leq s \leq 0 \\ \varphi(t+s) & s \leq-t\end{cases}
$$

(b) If $A_{1}$ is the operator in $E$ defined by

$$
D\left(A_{1}\right)=\left\{\varphi \in E_{0} \mid \varphi^{\prime} \in E\right\}, A_{1} \varphi=-\varphi^{\prime}, \varphi \in D\left(A_{1}\right),
$$

then:

(b1) $A_{1}$ is m-accretive;

(b2) $J_{\lambda}{ }^{A_{1}} \varphi=J_{\lambda}{ }^{A_{0}}(\varphi-\varphi(0))+\left(1-e_{\lambda}\right) \varphi(0)$ for all $\varphi \in E, \lambda>0$;

(b3) $J_{\lambda}{ }^{A} \varphi=J_{\lambda}{ }^{A_{1}} \varphi+e_{\lambda}\left(J_{\lambda}{ }^{A} \varphi\right)(0)$ for all $\varphi \in R(I+\lambda A), \lambda>0, \lambda \gamma<1$. 
(It is at this point where (E3) is particularly crucial. For the finite delay case, i.e., $E=C([-R, 0] ; X)$ with sup-norm, and $B=0$, the result goes back to [25]. For the general case of (E1)-(E3), cf. 70, Lemma 2.2].)

Now, let $\psi \in \hat{E}_{0}$. Then, as $\psi(0) \in \operatorname{cl}(D(B) \cap \hat{X})$, we can choose sequences $\left(x_{n}\right)_{n} \in D(B) \cap \hat{X},\left(y_{n}\right)_{n} \in B x_{n}$, and $\lambda_{n} \rightarrow 0^{+}, \lambda_{n} C_{E} L_{F}<1$, such that

$$
\left\|x_{n}-\psi(0)\right\| \rightarrow 0, \quad \lambda_{n}\left\|y_{n}\right\| \rightarrow 0, \quad \text { and } \quad \lambda_{n}\left\|F\left(\psi-\left(\psi(0)-x_{n}\right)\right)\right\| \rightarrow 0
$$

as $n \rightarrow \infty$. Invoking Lemma 3.1 for $\rho=\psi,(x, y)=\left(x_{n}, y_{n}\right) \in B$, and $\lambda=\lambda_{n}$ yields a sequence $\left(\varphi_{n}\right)_{n} \in D(A)$ with

$$
\varphi_{n}-\lambda_{n} \varphi_{n}^{\prime}=\psi+x_{n}+\lambda_{n} y_{n}-\left(\psi(0)+\lambda_{n} F \varphi_{n}\right), \quad \varphi_{n}(0)=x_{n} .
$$

Claim. $\left\|\psi-\varphi_{n}\right\| \rightarrow 0$.

Proof. First, notice that $\varphi_{n}=J_{\lambda_{n}}^{A}\left(\psi+x_{n}+\lambda_{n} y_{n}-\left(\psi(0)+\lambda_{n} F \varphi_{n}\right)\right)$. Invoking Lemma 3.2(b), for all $n \in \mathbb{N}$,

$$
\begin{aligned}
\| \psi & -\varphi_{n}\|=\| \psi-J_{\lambda_{n}}^{A}\left(\psi+x_{n}+\lambda_{n} y_{n}-\left(\psi(0)+\lambda_{n} F \varphi_{n}\right)\right) \| \\
& =\left\|\psi-J_{\lambda_{n}}^{A_{0}}(\psi-\psi(0))-\left(1-e_{\lambda_{n}}\right)\left(x_{n}+\lambda_{n} y_{n}-\lambda_{n} F \varphi_{n}\right)-e_{\lambda_{n}} x_{n}\right\| \\
& \leq\left\|(\psi-\psi(0))-J_{\lambda_{n}}^{A_{0}}(\psi-\psi(0))\right\|+\left\|\widetilde{\psi(0)}-\tilde{x_{n}}\right\|+\lambda_{n}\left\|\left(1-e_{\lambda_{n}}\right)\left(y_{n}-F \varphi_{n}\right)\right\| .
\end{aligned}
$$

We estimate the last term on the right of (3.18):

1. If we let $\rho_{n}:=\left(1-e_{\lambda_{n}}\right)\left(y_{n}-F \varphi_{n}\right)$, then $\rho_{n}-\lambda_{n} \rho_{n}^{\prime}=y_{n}-F \varphi_{n}$, and $\rho_{n}(0)=0$. Thus, according to (E2), in conjunction with (E1)(c),

$$
\left\|\left(1-e_{\lambda_{n}}\right)\left(y_{n}-F \varphi_{n}\right)\right\| \leq C_{E}\left\|y_{n}-F \varphi_{n}\right\| \leq C_{E}\left(\left\|y_{n}\right\|+\left\|F \varphi_{n}\right\|\right) .
$$

2. If we let $\rho_{n}:=\psi-\left(\psi(0)-x_{n}\right)$, then $\rho_{n} \in \hat{E}_{0}$, and $\rho_{n}(0)=x_{n}=\varphi_{n}(0)$. Thus, according to (A4) (b),

$$
\begin{aligned}
& \left\|F \varphi_{n}\right\| \leq\left\|F \varphi_{n}-F\left(\psi-\left(\psi(0)-x_{n}\right)\right)\right\|+\left\|F\left(\psi-\left(\psi(0)-x_{n}\right)\right)\right\| \\
& \leq \quad L_{F}\left\|\varphi_{n}-\left(\psi-\left(\psi(0)-x_{n}\right)\right)\right\|+\left\|F\left(\psi-\left(\psi(0)-x_{n}\right)\right)\right\| \\
& \leq \quad L_{F}\left(\left\|\varphi_{n}-\psi\right\|+C_{E}\left\|\psi(0)-x_{n}\right\|\right)+\left\|F\left(\psi-\left(\psi(0)-x_{n}\right)\right)\right\| .
\end{aligned}
$$

Invoking (3.19) and (3.20) into (3.18), and rearranging, yields

$$
\begin{aligned}
& \left(1-\lambda_{n} C_{E} L_{F}\right)\left\|\psi-\varphi_{n}\right\| \leq\left\|(\psi-\psi(0))-J_{\lambda_{n}}^{A_{0}}(\psi-\psi(0))\right\| \\
& +\quad C_{E}\left(1+\lambda_{n} C_{E} L_{F}\right)\left\|\psi(0)-x_{n}\right\|+\lambda_{n} C_{E}\left(\left\|y_{n}\right\|+\left\|F\left(\psi-\left(\psi(0)-x_{n}\right)\right)\right\|\right)
\end{aligned}
$$

for all $n \in \mathbb{N}$. According to (3.16), and taking into account that, by proposition (a) of Lemma 3.2, $J_{\lambda}^{A_{0}} \rho \rightarrow \rho$ as $\lambda \rightarrow 0^{+}$for all $\rho \in E_{0}$, we thus conclude that $\left\|\psi-\varphi_{n}\right\| \rightarrow 0$ as $n \rightarrow \infty$. This shows that $\psi \in \operatorname{cl} D(A)$ and completes the proof of (S3).

Taking into account that, at this point, we know that $\hat{E} \cap \operatorname{cl} D(A)=\hat{E}$, and thus that the semigroup $(S(t))_{t \geq 0}$ defined in (S2) actually acts on (all of) $\hat{E}$, proposition (S4) can be read from the proof of [58, Thm. 3.1]. (It is at this point where we particularly need both properties (d) (1) and (d) (2) of (E1) for the space E.) 
Step 4: Proof of (S5). Let $\varphi \in \hat{E}$. We have to show that, for any $T>0$, the function $u_{\varphi}(t):=(S(t) \varphi)(0), t \geq 0$, is a mild solution to the evolution equation

$$
(C P)_{\varphi} \quad\left\{\begin{array}{l}
\dot{u}(t)+B u(t) \ni F(S(t) \varphi), \quad 0 \leq t \leq T, \\
u(0)=\varphi(0) .
\end{array}\right.
$$

We shall need the following "normalization-result" for discrete-scheme approximations (as in (1.2), (1.3)) :

Given an operator $C \subset Z \times Z$ in a Banach space $Z, z_{0} \in Z, T>0$, and $f \in$ $L^{1}(0, T ; Z)$, if a continuous function $u:[0, T] \rightarrow Z$ with $u(0)=z_{0}$, is a mild solution on $[0, T]$ to

$$
(C P)\left\{C ; z_{0} ; f\right\} \quad\left\{\begin{array}{l}
\dot{u}(t)+C u(t) \ni f(t), 0 \leq t \leq T, \\
u(0)=z_{0}
\end{array}\right.
$$

then, for $\epsilon>0$, there exists an $\epsilon$-discrete-scheme approximation $D_{C}\left(t_{0}, \ldots, t_{N}\right.$; $\left.u_{0}, \ldots, u_{N} ; f_{1}, \ldots, f_{n}\right)$ with $t_{0}=0$, and $u_{0}=z_{0}$.

Proof. The possibility of normalizing one of the endpoints (here $t_{0}=0$ ) is known; cf. 7. Thm. 1.7,(ii)]. Thus, given any $\epsilon>0$, there exists an $\epsilon / 2$-discrete-scheme approximation $D_{C}\left(0, t_{1}, \ldots, t_{N} ; u_{0}, \ldots, u_{N} ; f_{1}, \ldots, f_{N}\right)$ to $(C P)\left\{C ; z_{0} ; f\right\}$. We now define $\tilde{u}_{0}=z_{0}$, and $\tilde{f}_{1}:=f_{1}+\left(1 / t_{1}\right)\left(u_{0}-z_{0}\right)$. Then $D_{C}\left(0, t_{1}, \ldots, t_{N} ; \tilde{u}_{0}, u_{1}, \ldots, u_{N}\right.$; $\left.\tilde{f}_{1}, f_{2}, \ldots, f_{N}\right)$ is an $\epsilon$-discrete-scheme approximation to $(C P)\left\{C ; z_{0} ; f\right\}$ of the desired form.

We proceed to show that $u_{\varphi}(t):=(S(t) \varphi)(0), t \geq 0$, is a mild solution to $(C P)_{\varphi}$ : Let $T>0$, and choose any sequence $\epsilon_{n} \searrow 0$, and recall that, by (S2), $S(\cdot) \varphi: \mathbb{R}^{+} \rightarrow E$ is a mild solution to

$$
(C P)\{A ; \varphi ; 0\}\left\{\begin{array}{l}
\dot{\phi}(t)+A \phi(t)=0, \quad t \geq 0 \\
\phi(0)=\varphi
\end{array}\right.
$$

Thus, letting $T_{1}=(T+1)$, say, given any $n \in \mathbb{N}$, there exists an $\epsilon_{n}$-discretescheme approximation $D_{A}\left(0, t_{1}^{n}, \ldots, t_{N_{n}}^{n} \leq(T+1) ; \varphi, \phi_{1}^{n}, \ldots, \phi_{N_{n}}^{n} ; \psi_{1}^{n}, \ldots, \psi_{N_{n}}^{n}\right)$ for $(C P)\{A ; \varphi ; 0\}$ on the interval $[0, T+1]$ (as in (1.2), (1.3) for $f=0$ ) such that, if $\Phi_{n}:\left[0, t_{N_{n}}^{n}\right] \rightarrow E$ is defined by

$$
\Phi_{n}(t)= \begin{cases}\varphi & t=0 \\ \phi_{i}^{n} & t \in\left(t_{i-1}^{n}, t_{i}^{n}\right], i \in\left\{1, \ldots, N_{n}\right\},\end{cases}
$$

then $\left\|\Phi_{n}(t)-S(t) \varphi\right\|<\epsilon_{n}$ for all $t \in\left[0, t_{N_{n}}^{n}\right]$. In particular,

$$
\left\|\Phi_{n}(t)-S(t) \varphi\right\|<\epsilon_{n} \quad \text { for all } t \in[0, T], n \in \mathbb{N} .
$$

We claim that

$$
\lim _{n \rightarrow \infty}\left\|F\left(\Phi_{n}(t)\right)-F(S(t) \varphi)\right\|=0 \quad \text { uniformly over } t \in[0, T] .
$$

(It is at this point where we need the normalization $\phi_{0}^{n}=\varphi$ : it is one of the subtle points of this paper that $F$ be defined just on $\hat{E}_{0}$, as the examples we want to include do not have $F$ even defined on $\epsilon$-neighbourhoods of $\hat{E}_{0}$. Notice that, by definition, all $\phi_{i}^{n} \in D(A) \subset \hat{E}_{0}, i \in\left\{1, \ldots, N_{n}\right\}$, and that this now also is true for $\phi_{0}^{n}=\varphi \in \hat{E} \subset \hat{E}_{0}$.)

(3.22) can be seen as follows:

1. $F: \hat{E}_{0} \rightarrow X$ is continuous, and thus uniformly continuous on compact subsets of $\hat{E}_{0}$. 
2. The set $M_{\varphi}=\{S(t) \varphi \mid t \in[0, T]\} \cup\left\{\Phi_{n}(t) \mid t \in[0, T], n \in \mathbb{N}\right\}$ is relatively compact in $\hat{E}_{0}$. In fact, given $\eta>0$, there exists $n_{0} \in \mathbb{N}$ such that, by (3.23), the set $\left\{\Phi_{n}(t) \mid t \in[0, T], n \geq N_{0}\right\}$ is $\eta$-close (in the $E$-norm) to the compact set $S([0, T]) \varphi$. As all $\Phi_{n}, n \in \mathbb{N}$, are finite-valued this shows that, given any $\eta>0$, the set $M_{\varphi}$ is $\eta$-close (in the $E$-norm) to the compact set $S([0, T]) \varphi \cup\left\{\Phi_{n}(t) \mid t \in\right.$ $\left.[0, T], n \in\left\{1, \ldots, n_{0}\right\}\right\} \subset \hat{E}_{0}$, and thus is relatively compact. (Notice that, as $\hat{E}_{0}$ is closed in $E$, the $E$-closure of $M_{\varphi}$ is (compact and) contained in $\hat{E}_{0}$.)

Results 1 and 2, in conjunction with (3.21), serve to prove (3.22).

Thus, we can choose $n_{0}$ large enough such that both $\epsilon_{n_{0}}<\epsilon / 2$ and

$$
\int_{0}^{T}\left\|F\left(\Phi_{n_{0}}(\tau)\right)-F(S(\tau) \varphi)\right\| d \tau<\epsilon / 2
$$

We then cut off the $\epsilon_{n_{0}}$-discretization of $[0, T+1]$ corresponding to $\Phi_{n_{0}}$ at the point $t_{i_{0}}^{n_{0}}$ for which $t_{i_{0}}^{n_{0}} \leq T<t_{i_{0}+1}^{n_{0}}$, and let $N=i_{0}$. Suppressing, in the following, the upper index $n_{0}$ everywhere, we thus have an $\epsilon_{n_{0}}$-partition of the interval $[0, T]$,

$$
0=t_{0}<t_{1}<\ldots<t_{N} \leq T, t_{i}-t_{i-1}<\epsilon_{n_{0}}, i \in\{1, \ldots, N\}, T-t_{N}<\epsilon_{n_{0}},
$$

and elements $\left\{\phi_{0}=\varphi, \phi_{1}, \ldots, \phi_{N}\right\}$, and $\left\{\psi_{1}, \ldots, \psi_{N}\right\}$ in $E$ such that

$$
\begin{aligned}
& \phi_{i} \in D(A), i \in\{1, \ldots, N\}, \\
& \frac{\phi_{i}-\phi_{i-1}}{t_{i}-t_{i-1}}+A \phi_{i}=\psi_{i}, i \in\{1, \ldots, N\}, \\
& \sum_{1}^{N}\left(t_{i}-t_{i-1}\right)\left\|\psi_{i}\right\|<\epsilon_{n_{0}},
\end{aligned}
$$

and such that, if the step function $\tilde{\Phi}_{n_{0}}:\left[0, t_{N}\right] \rightarrow E$ is defined by

$$
\tilde{\Phi}_{n_{0}}(t)=\left.\Phi_{n_{0}}\right|_{\left[0, t_{N}\right]}(t)= \begin{cases}\varphi & t=0, \\ \phi_{i} & t \in\left(t_{i-1}, t_{i}\right], i \in\{1, \ldots, N\},\end{cases}
$$

then $\left\|\tilde{\Phi}_{n_{0}}(t)-S(t) \varphi\right\|<\epsilon_{n_{0}}$ uniformly over $t \in\left[0, t_{N}\right]$.

Let $u_{i}=\phi_{i}(0), i \in\{0,1, \ldots, N\}$, and $f_{i}=\psi_{i}(0)+F\left(\phi_{i}\right), i \in\{1, \ldots, N\}$. Then, recalling from the definition of $D(A)$ that $A \phi_{i}(0)=-\phi_{i}^{\prime}(0) \in B\left(\phi_{i}(0)\right)-F\left(\phi_{i}\right), i \in$ $\{1, \ldots, N\}$, we read from (3.25) and (3.23) that

$$
\begin{aligned}
& u_{i} \in D(B), i \in\{1, \ldots, N\}, \\
& \frac{u_{i}-u_{i-1}}{t_{i}-t_{i-1}}+B u_{i} \ni f_{i}, i \in\{1, \ldots, N\}, \\
& \sum_{1}^{N} \int_{t_{i-1}}^{t_{i}}\left\|f_{i}-F(S(\tau) \varphi)\right\| d \tau=\sum_{1}^{N} \int_{t_{i-1}}^{t_{i}}\left\|\psi_{i}(0)+F\left(\phi_{i}\right)-F(S(\tau) \varphi)\right\| d \tau \\
\leq & \sum_{1}^{N}\left(t_{i}-t_{i-1}\right)\left\|\psi_{i}\right\|+\int_{0}^{t_{N}}\left\|F\left(\tilde{\phi}_{n_{0}}(\tau)\right)-F(S(\tau) \varphi)\right\| d \tau<\epsilon_{n_{0}}+\epsilon / 2,
\end{aligned}
$$

and such that, if the step function $u_{\epsilon}:\left[0, t_{N}\right] \rightarrow X$ is defined by

$$
u_{\epsilon}(t)= \begin{cases}u_{0} & t=0, \\ u_{i} & t \in\left(t_{i-1}, t_{i}\right], i \in\{1, \ldots, N\},\end{cases}
$$


then $u_{\epsilon}(t)=\tilde{\Phi}_{n_{0}}(t)(0), t \in\left[0, t_{N}\right]$; hence $\left\|u_{\epsilon}(t)-(S(t) \varphi)(0)\right\| \leq\left\|\tilde{\Phi}_{n_{0}}(t)-S(t) \varphi\right\|$ $<\epsilon_{n_{0}}$ uniformly over $t \in\left[0, t_{N}\right]$. Recalling now that we have chosen $\epsilon_{n_{0}}<\epsilon / 2$, we thus have arrived at the desired $\epsilon$-approximate solution $u_{\epsilon}$ of $u_{\varphi}=(S(\cdot) \varphi)(0)$ on $\left[0, t_{N}\right]$ as in (1.2)-(1.3). This completes the proof of (S5).

Recalling that the estimate (2.2) is the usual integral inequality for the difference of two mild solutions to the Cauchy problem governed by an $\omega$-accretive operator (cf. 7, 48), the proof of propositions (a) and (c) of Theorem 2.1 is thus complete.

Proposition (b) of Theorem 2.1, in turn, is an easy consequence of (2.2) and (E3): Given either of the extra assumptions of proposition (b), assume that, given $\psi \in \hat{E}$, there exist mild solutions $u, v$ to (PFDE) with $u_{0}=v_{0}=\psi, u_{t}, v_{t} \in \hat{E}$ for all $t \geq 0$, and $u \neq v$. Let $t_{0}:=\sup \{t \geq 0 \mid u(s)=v(s)$ for all $0 \leq s \leq t\}$. Then $0 \leq t_{0}<\infty$, and, by continuity of $u, v, u\left(t_{0}\right)=v\left(t_{0}\right)$, and $u_{s}=v_{s}$ for all $s \in\left[0, t_{0}\right]$. According to either of the extra assumptions, there exist $\delta_{0}>0$ and $M_{0}>0$ such that $\left[\psi_{1}(0)-\psi_{2}(0), F \psi_{1}-F \psi_{2}\right] \leq M_{0}\left\|\psi_{1}-\psi_{2}\right\|$ for all $\psi_{1}, \psi_{2} \in \hat{E}$ with $\left\|\psi_{i}-u_{t_{0}}\right\|<\delta_{0}, i \in\{1,2\}$. According to (E3)(b), $K:=\sup \left\{M_{1}(s) \mid 0 \leq\right.$ $\left.s \leq t_{0}+\delta_{0}\right\}<\infty$. Choose $0<\delta<\delta_{0}$ such that $\delta M_{0} K e^{(|\omega|+\omega)\left(t_{0}+\delta_{0}\right)}<1$, and $\left\|u_{\tau}-u_{t_{0}}\right\|,\left\|v_{\tau}-u_{t_{0}}\right\|<\delta_{0}$ for all $\tau \in\left[t_{0}, t_{0}+\delta\right]$. Next, choose $t_{1} \in\left(t_{0}, t_{0}+\delta\right]$ such that $\left\|u\left(t_{1}\right)-v\left(t_{1}\right)\right\| \geq\|u(s)-v(s)\|$ for all $s \in\left[t_{0}, t_{0}+\delta\right]$. From (E3)(b), in conjunction with (2.2), we conclude

$$
\begin{aligned}
& \left\|u\left(t_{1}\right)-v\left(t_{1}\right)\right\| \leq e^{\omega t_{1}} M_{0} \int_{t_{0}}^{t_{1}} e^{-\omega \tau}\left\|u_{\tau}-v_{\tau}\right\| d \tau \\
& \leq M_{0} K\left\|u\left(t_{1}\right)-v\left(t_{1}\right)\right\| e^{\omega t_{1}} \int_{t_{0}}^{t_{1}} e^{|\omega| \tau} d \tau \leq \delta M_{0} K e^{(|\omega|+\omega) t_{1}}\left\|u\left(t_{1}\right)-v\left(t_{1}\right)\right\| \\
& <\left\|u\left(t_{1}\right)-v\left(t_{1}\right)\right\| .
\end{aligned}
$$

This contradiction serves to prove Theorem 2.1 (b), and the proof of Theorem 2.1 is complete.

3.2. Proof of Remark 2.2, part 1. Given assumption $(2.3)$, let $x \in(\operatorname{cl} D(B)) \cap$ $\hat{X}$. Then, using (3.9), $\left\|x-J_{\lambda}{ }^{B} x\right\| \leq\left(1+(1-\lambda \omega)^{-1}\right)\left\|x-x_{0}\right\|+\lambda(1-\lambda \omega)^{-1}\left\|y_{0}\right\|$ for all $\left(x_{0}, y_{0}\right) \in B$, and all $\lambda>0$ small enough. Thus, $\limsup _{\lambda \rightarrow 0^{+}}\left\|x-J_{\lambda}{ }^{B} x\right\| \leq$ $2\left\|x-x_{0}\right\|$ for all $x_{0} \in D(B)$. As $x \in \operatorname{cl} D(B)$, and, by assumption, $J_{\lambda}{ }^{B} x \in D(B) \cap$ $\hat{X}$, this serves to prove that $x \in \operatorname{cl}(D(B) \cap \hat{X})$, and thus completes the proof.

3.3. Proof of Theorem 2.5. As already noted prior to the formulation of Theorem 2.5, the idea of the proof is from [9, Lemma 4.2] for the nondelay case: Given $\psi \in \hat{E}$, and $\epsilon>0$, assumption (2.5) of Theorem 2.5 implies that there exist $0<\lambda<$ $\epsilon$, and $x_{\lambda} \in \hat{X}$ such that $\left\|\psi(0)+\lambda F(\psi)-x_{\lambda}\right\|<\lambda \epsilon$. Let $\psi(0)+\lambda F(\psi)-x_{\lambda}=: e_{\lambda}$. Then $\left\|e_{\lambda}\right\|<\lambda \epsilon$, and $\psi(0)+\lambda F(\psi)-e_{\lambda}=x_{\lambda} \in \hat{X}$. Thus, according to assumption (2.4) of Theorem 2.5, $J_{\lambda}^{B}\left(\psi(0)+\lambda F(\psi)-e_{\lambda}\right)=J_{\lambda}{ }^{B}\left(x_{\lambda}\right) \in D(B) \cap \hat{X}$. Hence,

$$
\begin{aligned}
& \psi(0)+\lambda F(\psi)-e_{\lambda} \in(I+\lambda B)(D(B) \cap \hat{X}), \quad \text { and thus } \\
& \operatorname{dist}(\psi(0)+\lambda F(\psi) ;(I+\lambda B)(D(B) \cap \hat{X})) \leq\left\|e_{\lambda}\right\|<\lambda \epsilon .
\end{aligned}
$$

This shows that condition (STC) of Theorem 2.1 holds. The final statement on $\hat{E}_{0}$ is a consequence of assumption (2.4) in conjunction with Remark 2.2. This completes the proof of Theorem 2.5. 
3.4. Proof of Lemma 2.7. From Step 2.1 of the proof of Theorem 2.1, notably (3.11), we know that $\varphi_{\lambda, \psi(0)}^{\psi} \rightarrow \psi$ as $\lambda \searrow 0$. By assumption, for $\lambda>0$ small enough, there exist $x_{\lambda} \in D(B) \cap \hat{X}$, and $y_{\lambda} \in B x_{\lambda}$ such that $\psi(0)+\lambda F\left(\varphi_{\lambda, \psi(0)}^{\psi}\right)=x_{\lambda}+\lambda y_{\lambda}$. Thus,

$$
\begin{aligned}
& \frac{1}{\lambda} \operatorname{dist}(\psi(0)+\lambda F \psi ;(I+\lambda B)(D(B) \cap \hat{X})) \\
& \leq \frac{1}{\lambda}\left[\left\|\psi(0)+\lambda F\left(\varphi_{\lambda, \psi(0)}^{\psi}\right)-\left(x_{\lambda}+\lambda y_{\lambda}\right)\right\|+\lambda\left\|F \psi-F\left(\varphi_{\lambda, \psi(0)}^{\psi}\right)\right\|\right] \\
& =\left\|F \psi-F\left(\varphi_{\lambda, \psi(0)}^{\psi}\right)\right\| \rightarrow 0 \text { as } \lambda \searrow 0 .
\end{aligned}
$$

This completes the proof.

Finally, we note that if (A4) (c) is strengthened to $\left\|F \varphi_{1}-F \varphi_{2}\right\| \leq M_{F}\left\|\varphi_{1}-\varphi_{2}\right\|$, and (LRC) is fulfilled, then $(I+\lambda A)(D(A) \cap \hat{E}) \supset \hat{E}$ (compare the method of proof of 70. Theorem 2.1,(a)]), which, trivially, implies the subtangential condition (STC) $A$ (of the discussion preceding the proof of Theorem 2.1 above) for $A$, "usually" guaranteeing flow-invariance of $\hat{E}$. For this aspect of (A4) (c), though, see Remark 5.6 below.

\section{LiNEARIZED STABILITY AND REGULARITY}

In this section, we formulate and discuss two results on linearized stability, and on regularity of solutions to (PFDE). We shall restrict ourselves to the case of $B \subset X \times X$ linear $\omega-\mathrm{m}$-accretive. The general nonlinear case requires additional concepts and techniques and will be treated elsewhere.

The following notions of (relative) Fréchet-differentiability of a nonlinear map from $X$ to $Y, X$ and $Y$ Banach spaces, will be basic for our considerations.

Definition 4.1. 1. A mapping $H: D(H) \subset X \rightarrow Y$ is said to be $\mathrm{F}$-differentiable at $x \in D \subset D(H)$ (relative to $D$ ) if the following holds: There exists $\tilde{H}[x] \in B(X, Y)$ such that, given any $\epsilon>0$, there exists $\delta>0$ such that, if $z \in D$, and $\|z-x\|<\delta$, then

$$
\|H z-H x-\tilde{H}[x](z-x)\| \leq \epsilon\|z-x\|
$$

2. A mapping $H: D(H) \subset X \rightarrow Y$ is said to be continuously F-differentiable on $D \subset D(H)$ if it is F-differentiable at each $x \in D$ (in the sense of 1, above), and if the map $\{x \rightarrow \tilde{H}[x]\}$ is continuous from $D$ to $B(X, Y)$.

(Here, $B(X, Y)$ denotes the space of bounded linear operators from $X$ to $Y$ with operator norm.) Notice that, in contrast to classical Fréchet-differentiability, the approximation is only required on a relative neighbourhood with respect to $D \subset D(H)$ (as even $D(H)$, in general, may not contain any $X$-open subset).

The principle of linearized stability, roughly, is about the question of whether an equilibrium of a nonlinear differential equation is locally exponentially stable, provided the "linearization" around the equilibrium is exponentially stable. The answer in the context of problem (PFDE) is the following.

Theorem 4.2. Under the general assumptions of Theorem 2.1, let, in addition, $B \subset X \times X$ be linear and $\omega-m$-accretive, and assume that there exists an equilibrium solution to (PFDE), i.e., an element $\varphi_{e} \in \hat{E} \cap D(A)$ such that $S(t) \varphi_{e} \equiv \varphi_{e}$. If the operator $\left.F\right|_{\hat{E}}: \hat{E} \rightarrow X$ has an $F$-derivative $\tilde{F}\left[\varphi_{e}\right] \in B(E, X)$ at $\varphi_{e}$ (in the sense 
of Definition 4.1,1.), and if the solution semigroup $(\tilde{S}(t))_{t \geq 0}$ of the corresponding linear $(P F D E)_{l i n}$, with $F$ being replaced by $\tilde{F}\left[\varphi_{e}\right]$, is exponentially asymptotically stable, then the equilibrium $\varphi_{e}$ is locally exponentially stable for the original (nonlinear) (PFDE). More precisely, there exist $\delta>0, K \geq 1$, and $\alpha>0$ such that for all $\psi \in \hat{E}$ with $\left\|\psi-\varphi_{e}\right\|<\delta$,

$$
\left\|u_{\psi}(t)-\varphi_{e}(0)\right\| \leq\left\|S(t) \psi-\varphi_{e}\right\| \leq K e^{-\alpha t}\left\|\psi-\varphi_{e}\right\| \quad \text { for all } t \geq 0 .
$$

Remarks 4.3. 1. In the global case $\hat{X}=X, \hat{E}=E$, and $F: E \rightarrow X$ globally Lipschitz, versions of Theorem 4.2 have been proved (a) in the finite-delay case for $B: D(B) \subset X \rightarrow X$ (linear and) single-valued under the assumption that $F$ be globally Fréchet-differentiable with $F^{\prime}: E \rightarrow B(E, X)$ being locally Lipschitz (36, Thm. 6.1], [52, 53]), and (b) in the infinite-delay case for $B: D(B) \subset X \rightarrow X$ (single-valued and) a Hille-Yosida operator (1]), and $F$ Fréchet-differentiable at the equilibrium. (We repeat, once again, that Hille-Yosida operators are (single-valued, linear and) $\omega-\mathrm{m}$-accretive for an equivalent norm.)

2. Under local range flow-invariance conditions for $\hat{X} \subset X$, and $\hat{E} \subset E$, versions of Theorem 4.2 have been proved in [72, Thm. 2.4] for $B: D(B) \subset X \rightarrow X$ the infinitesimal generator of a $C_{0}$-semigroup of bounded linear operators on $X$, and in [68, Thm. 4.1] for $B \subset X \times X \omega$-accretive, possibly nonlinear, under additional "resolvent-differentiability" conditions on $B$ (for details, see [68]).

As Theorem 4.1 touches on exponential asymptotic stability of the solution semigroup to (a linearization of) (PFDE), we pause for a moment to discuss this question.

In its generality, even if $\left(\omega+M_{F}\right)<0$, i.e., if the damping exerted by the accretivity of $B$ dominates the influence of the history, Theorem 2.1 does not assert exponential stability, since the stability constant for $(S(t))_{t \geq 0}$ is given by $\gamma=$ $\max \left\{0, \omega+M_{F}\right\}$. However, under specific assumptions, this general result can be improved to yield exponential stability.

Proposition 4.4. Under the general assumptions of Theorem 2.1, assume, in addition, that $\left(\omega+M_{F}\right)<0$. If either

(a) $I=[-R, 0]$ (finite delay), and $E=C([-R, 0] ; X)$ with sup-norm, or

(b) $I=(-\infty, 0]$ (infinite delay), and $E$ is an $E_{v}-$ space with weight $v$ such that the function $\left\{s \rightarrow v(s) e^{-\mu s}\right\}, s \leq 0$, is nondecreasing for some $\mu>0$,

then the solution semigroup $(S(t))_{t \geq 0}$ to $(P F D E)$ on $\hat{E}$ is exponentially stable; more specifically, there exists $K \geq 1$ such that

$$
\left\|S(t) \psi_{1}-S(t) \psi_{2}\right\| \leq K e^{-\beta t}\left\|\psi_{1}-\psi_{2}\right\| \quad \text { for all } \psi_{1}, \psi_{2} \in \hat{E} \text {, and all } t \geq 0,
$$

where $\beta$ can be chosen as $\beta=-\left(\omega+M_{F}\right)$ in case (a), and $\beta=\min \left\{\mu,-\left(\omega+M_{F}\right)\right\}$ in case (b).

Remark. The finite-delay part of case (a) can be traced back to [59. The results in both cases (a) and (b) can be read from the technique of the proof of [71, Thm. 3.6], with obvious minor modifications (as in [71, beyond our assumption (A4), F was assumed to be Lipschitz on $\hat{E}$ ). Altogether, for a thorough discussion of, and further results on stability properties of (PFDE), the reader is referred to 63, 64, [67, Section 3] and [71]. 
Turning to regularity of solutions in the context of Theorem 2.1, there are obvious positive results for geometrically well-behaved state spaces: if $X$ has the RadonNikodym Property (RNP), and $B$ is $\omega$-m-accretive, and if $\psi \in \hat{E} \cap \hat{D}(A)$, then the solution $u_{\psi}$ as in Theorem 2.1 is a strong solution to (PFDE); i.e., it is differentiable a.e. $t \geq 0$, and fulfills (PFDE) pointwise a.e. $t \geq 0$. $(\hat{D}(A)$ is the generalized domain of $A$.) The result follows by combining the results of [15] and [7, Cor. 7.5] with $X$ having the RNP. However, two of the most interesting state spaces, $C(\bar{\Omega})$ for $\Omega$ bounded open in $\mathbb{R}^{N}$, and $L^{1}(\Omega)$ for $\Omega$ open in $\mathbb{R}^{N}$, do not enjoy the RNP. For general Banach spaces, even for the special case of the linear Cauchy problem $\dot{u}(t)+A u(t)=f(t), u_{0} \in D(A)$, with $-A$ the generator of a $C_{0}$-semigroup of bounded linear operators on $X$, the regularity of the solutions depends on the regularity properties of $f$ - such as local uniform Hölder continuity of $f$ with $A$ parabolic, or continuous differentiability of $f$ for general $A$.

It is thus natural to ask whether the differentiability conditions on $F$ are sufficient to ensure the regularity of solutions to (PFDE). Notice that this is not a priori clear, as the inhomogeneity $f$ in the case of (PFDE) implicitly includes the solution semigroup $(S(t))_{t \geq 0}: f(t)=F(S(t) \psi)$. Nevertheless, our result on the regularity of solutions to (PFDE) solves the problem in the affirmative.

Theorem 4.5. Under the general assumptions of Theorem 2.1, let, in addition, $B \subset X \times X$ be linear and $\omega-m$-accretive, and assume that $\hat{E}$ is convex. Moreover, assume that $F$ is continuously $F$-differentiable on $\hat{E}$ (in the sense of Definition 4.1, 2, above). Then, for all $\varphi \in \hat{E} \cap D(A)$ with $\varphi^{\prime}(0) \in \operatorname{cl} D(B)$, and with u$\varphi$ denoting the corresponding mild solution to (PFDE) as in Theorem 2.1, the following hold:

(i) $u_{\varphi}: \mathbb{R}^{+} \rightarrow X$ is continuously differentiable, and is a classical solution to (PFDE), i.e. fulfills (PFDE) for all $t \geq 0$.

(ii) $\left\|\frac{d}{d t} u_{\varphi}(t)\right\| \leq e^{\tilde{\gamma}(t) t}\left\|\varphi^{\prime}\right\|$ for all $t \geq 0$, with $\tilde{\gamma}(t):=\max \{0, \omega+\gamma(t)\}$, and $\gamma(t)=\max \{\|\tilde{F}[S(s) \varphi]\| \mid 0 \leq s \leq t\}$.

(iii) The mapping $\left\{t \rightarrow \frac{d}{d t} u_{\varphi}(t)\right\}$ is a mild solution to the linearized nonautonomous version of (PFDE):

$$
(P F D E)_{l i n}\left\{\begin{array}{l}
\dot{v}(t)+B v(t) \ni \tilde{F}[S(t) \varphi] v_{t}, t \geq 0, \\
v_{\mid I}=\varphi^{\prime} .
\end{array}\right.
$$

Remarks 4.6. 1. The regularity result of Theorem 4.5 (i), so far, has been proved for the global case of $\hat{X}=X, \hat{E}=E$, and $F: E \rightarrow X$ globally Lipschitz, with $B$ : $D(B) \subset X \rightarrow X$ (single-valued) linear and either (a) generating a $C_{0}$-semigroup of bounded linear operators on $X$ ([76, Prop. 2.3]), or (b) a Hille-Yosida operator in $X([1,23)$, yet under the further stronger hypothesis of $F: E \rightarrow X$ being globally Fréchet-differentiable, with the mapping $F^{\prime}: E \rightarrow B(E, X)$ being locally Lipschitz. 2 . The case $B \equiv 0$ : While the existence and flow-invariance results of section 2, specialized to $B \equiv 0$, are just complements to existing results for ordinary functional differential equations, the linearized stability and regularity results of Theorems 4.2 and 4.5 seem to be new even for this special case.

Proofs of Theorems 4.2 and 4.5. According to the assumptions in Theorems 4.2 and 4.5 , for the remainder of this section, we assume that

(A1)* $B \subset X \times X$ is linear and $\omega-\mathrm{m}$-accretive. 
The proofs of Theorems 4.2 and 4.5 will be based on the subsequent technical result.

Lemma 4.7. Under the assumptions of Theorem 2.1, with (A1) being specialized to $(\mathbf{A 1})^{*}$, and given $T>0$, let $\varphi \in \hat{E}$ such that $\left.F\right|_{\hat{E}}: \hat{E} \rightarrow X$ has an $F$-derivative $\tilde{F}[S(t) \varphi] \in B(E, X)$ at $S(t) \varphi$ for all $t \in[0, T]$ (in the sense of Definition 4.1,1), with the map $\{t \rightarrow \tilde{F}[S(t) \varphi]\}$ continuous from $[0, T]$ to $B(E, X)$. Also, consider the family $\{\tilde{A}(t) \mid 0 \leq t \leq T\}$ of linear operators in $E$, defined by

$$
\left\{\begin{array}{l}
D(\tilde{A}(t))=\left\{\rho \in E \mid \rho^{\prime} \in E, \rho(0) \in D(B), \rho^{\prime}(0) \in \tilde{F}[S(t) \varphi] \rho-B \rho(0)\right\} \\
\tilde{A}(t) \rho:=-\rho^{\prime}, \rho \in D(\tilde{A}(t))
\end{array}\right.
$$

Then the following hold:

(a) The family $\{\tilde{A}(t) \mid 0 \leq t \leq T\}$ generates a linear evolution system $\{U(t, 0) \mid$ $0 \leq t \leq T\}$ in $E$ such that, if $\rho \in E$ with $\rho(0) \in \operatorname{cl} D(B)$, the function $\tilde{u}_{\rho}: I \cup \mathbb{R}^{+} \rightarrow$ $X$, defined by

$$
\tilde{u}_{\rho}(t)= \begin{cases}\rho(t) & t \in I \\ (U(t, 0) \rho)(0) & t \geq 0\end{cases}
$$

is a mild solution to the linearized nonautonomous version of (PFDE),

$$
(P F D E)_{l i n}\left\{\begin{array}{l}
\dot{v}(t)+B v(t) \ni \tilde{F}[S(t) \varphi] v_{t}, \quad 0 \leq t \leq T \\
v_{\left.\right|_{I}}=\rho
\end{array}\right.
$$

and $U(t, 0) \rho=\left(\tilde{u}_{\rho}\right)_{t}, 0 \leq t \leq T$.

(b) There exist bounded functions $C_{1}, C_{2}:[0, T] \rightarrow \mathbb{R}^{+}$such that, for all $\psi \in \hat{E}$,

$$
\begin{aligned}
& \|S(t) \psi-S(t) \varphi-U(t, 0)(\psi-\varphi)\| \\
& \quad \leq C_{1}(t) e^{C_{2}(t)} \int_{0}^{t}\|F(S(\tau) \psi)-F(S(\tau) \varphi)-\tilde{F}[S(\tau) \varphi](S(\tau) \psi-S(\tau) \varphi)\| d \tau
\end{aligned}
$$

for all $t \in[0, T]$,

Proof. Part (a), in a sense, is a combination of known results on the nonautonomous version of (PFDE); cf. [19, 20, 66]. Let $\gamma(T)=\max \{\|\tilde{F}[S(t) \varphi]\| \mid 0 \leq t \leq$ $T\}$, and $\tilde{\gamma}(T):=\max \{0, \omega+\gamma(T)\}$. Then, according to [70, Thm. 2.1, Prop. 2.7], all operators $\tilde{A}(t)$ are $\tilde{\gamma}(T)$-m-accretive in $E$, with $c l D(\tilde{A}(t))=\{\rho \in E$ $\rho(0) \in \operatorname{cl} D(B)\}$ independent of $t \in[0, T]$. Also, as, according to (E2), given $t, \tau \in[0, T],\left\|J_{\lambda}^{\tilde{A}(t)} \rho-J_{\lambda}^{\tilde{A}(\tau)} \rho\right\|=\left\|\left(J_{\lambda}^{\tilde{A}(t)} \rho\right)(0)-\left(J_{\lambda}^{\tilde{A}(\tau)} \rho\right)(0)\right\|$, an easy computation reveals that

$$
\left\|J_{\lambda}^{\tilde{A}(t)} \rho-J_{\lambda}^{\tilde{A}(\tau)} \rho\right\| \leq \frac{\lambda}{(1-\lambda \tilde{\gamma}(T))(1-\lambda \gamma(T))}\|\tilde{F}[S(t) \varphi]-\tilde{F}[S(\tau) \varphi]\|\|\rho\|
$$

for all $\rho \in E$, and all $\lambda>0$ small enough. Thus, according to [16, Thm. 2.1], the family $\{\tilde{A}(t) \mid 0 \leq t \leq T\}$ generates an evolution system $\{U(t, 0) \mid 0 \leq t \leq T\}$ of bounded linear operators from $D:=\{\rho \in E \mid \rho(0) \in \operatorname{cl} D(B)\}$ into $D$. The fact that $U(t, 0) \rho=\left(\tilde{u}_{\rho}\right)_{t}, t \in[0, T]$, for all $\rho \in D$ follows from [58, Thm. 3.1] (where we take advantage of the fact that the $f_{i}$ 's in the proof of this result can all be taken to be the zero-functions, as, in our case, all operators $\tilde{A}(t)$ are $\tilde{\gamma}(T)$-m-accretive). The (possibly) only new fact beyond the papers [19, 20, 33, 34, 62, 66] is the result that, for $\rho \in D,(U(\cdot, 0) \rho)(0)$ is a mild solution to (4.3). This, however, can be 
shown as in the corresponding autonomous case above (Step 4, proof of (S5) in section 3), or can be read from the corresponding proof for the more general result of [26, Thm. 3.3].

As for Lemma 4.7 (b), we start from the observation that, as $B \subset X \times X$ is linear, if $u_{\varphi}$ and $u_{\psi}$ denote the mild solutions to (PFDE) as in Theorem 2.1 for the initial histories $\varphi$ and $\psi$, respectively, then $\left(u_{\psi}-u_{\varphi}\right)$ is a mild solution to

$$
\left\{\begin{array}{l}
\dot{w}(t)+B w(t) \ni F(S(t) \psi)-F(S(t) \varphi), \quad 0 \leq t \leq T \\
w_{\mid I}=\psi-\varphi
\end{array}\right.
$$

Moreover, from (a), the function $(U(\cdot, 0)(\psi-\varphi))(0)$ is a mild solution to

$$
\left\{\begin{array}{l}
\dot{z}(t)+B z(t) \ni \tilde{F}[S(t) \varphi] z_{t}, \quad 0 \leq t \leq T, \\
z_{\mid I}=\psi-\varphi
\end{array}\right.
$$

Thus, by the integral inequality for mild solutions for $B \omega$-accretive ([7, Ch. 6]),

$$
\begin{aligned}
& e^{-\omega t}\left\|u_{\psi}(t)-u_{\varphi}(t)-(U(t, 0)(\psi-\varphi))(0)\right\| \\
& \leq \int_{0}^{t} e^{-\omega \tau}\|F(S(\tau) \psi)-F(S(\tau) \varphi)-\tilde{F}[S(\tau) \varphi](U(\tau, 0)(\psi-\varphi))\| d \tau
\end{aligned}
$$

for all $t \in[0, T]$. In particular,

$$
\begin{aligned}
& \max _{0 \leq s \leq t}\left\|u_{\psi}(s)-u_{\varphi}(s)-(U(s, 0)(\psi-\varphi))(0)\right\| \\
& \leq e^{2|\omega| t} \int_{0}^{t}\|F(S(\tau) \psi)-F(S(\tau) \varphi)-\tilde{F}[S(\tau) \varphi](U(\tau, 0)(\psi-\varphi))\| d \tau
\end{aligned}
$$

for all $t \in[0, T]$. We now define the function $x: I \cup \mathbb{R}^{+} \rightarrow X$ by $x(s)=0$ for $s \leq 0$, and $x(s)=u_{\psi}(s)-u_{\varphi}(s)-(U(s, 0)(\psi-\varphi))(0)$ for $s \geq 0$. An easy calculation (using, in particular, the translation properties of $S(\cdot)$ and $U(\cdot, 0))$ then shows that, for $t \in[0, T]$, the function $x_{t}: I \rightarrow X$ is given by $x_{t}=S(t) \psi-S(t) \varphi-U(t, 0)(\psi-\varphi)$. Thus, according to (E3) (b), as $x_{0} \equiv 0,(4.5)$ implies

$$
\begin{aligned}
& \|S(t) \psi-S(t) \varphi-U(t, 0)(\psi-\varphi)\| \\
& \leq \quad M_{1}(t) e^{2|\omega| t} \int_{0}^{t}\|F(S(\tau) \psi)-F(S(\tau) \varphi)-\tilde{F}[S(\tau) \varphi](U(\tau, 0)(\psi-\varphi))\| d \tau \\
& \leq \quad M_{1}(t) e^{2|\omega| t} \int_{0}^{t}\|F(S(\tau) \psi)-F(S(\tau) \varphi)-\tilde{F}[S(\tau) \varphi](S(\tau) \psi-S(\tau) \varphi)\| d \tau \\
& +\quad M_{1}(t) e^{2|\omega| t} \gamma(t) \int_{0}^{t}\|S(\tau) \psi-S(\tau) \varphi-U(\tau, 0)(\psi-\varphi)\| d \tau,
\end{aligned}
$$

with $\gamma(t)=\max \{\|\tilde{F}[S(s) \varphi]\| \mid 0 \leq s \leq t\}$. An application of Gronwall's Lemma completes the proof of Lemma 4.7.

Proof of Theorem 4.2. With $\varphi=\varphi_{e}$, and thus $U(\cdot, 0)=\tilde{S}(\cdot)$ the solution semigroup for the linearized (PFDE) (with $F$ being replaced by $\tilde{F}\left[\varphi_{e}\right]$ ), (4.4) of Lemma 4.7, in conjunction with the F-differentiability of $\left.F\right|_{\hat{E}}$ at $\varphi_{e}$, implies that, given any $t>0, \tilde{S}(t)$ is an F-differential of $S(t)$ at $\varphi_{e}$ (in the sense of Definition 4.1, part 1). As the semigroup $(\tilde{S}(t))_{t \geq 0}$ is supposed to be exponentially asymptotically stable, the result of Theorem 4.2 now follows from [18, Prop. 2.1]. (Originally, in [18, this 
result has been proved for semigroups defined on an open subset of a Banach space, with classical Fréchet-differentiability at the equilibrium. However, as explicitly reproduced in [69, Final Remarks 2], the original method of proof works just as well for any subset of a Banach space and F-differentiability at the equilibrium in the sense of Definition 4.1, part 1).

Proof of Theorem 4.5. Under the assumptions of Theorem 4.5, let $\varphi \in \hat{E} \cap D(A)$ such that $\varphi^{\prime}(0) \in \operatorname{cl} D(B)$.

Step 1: $u_{\varphi}^{\prime+}(0):=\lim _{t \rightarrow 0^{+}} \frac{1}{t}(u \varphi(t)-u(0))=\varphi^{\prime}(0)$.

Proof. As $\varphi^{\prime}(0) \in \operatorname{cl} D(B)$, there exists a unique mild solution $w$ to the Cauchy problem $\dot{w}(t)+B w(t) \ni 0, w(0)=\varphi^{\prime}(0)$. By linear theory, cf. [7, Thm. 5.1] and [17, Section 6], the function $W(t)=\int_{0}^{t} w(\tau) d \tau$ is a classical solution to $\dot{W}(t)+B W(t) \ni$ $\varphi^{\prime}(0), W(0)=0$. As $\varphi \in D(A)$, and thus $B \varphi(0) \ni F \varphi-\varphi^{\prime}(0)$, we conclude that $v(t):=W(t)+\varphi(0)$ is a mild solution to $\dot{v}(t)+B v(t) \ni \varphi^{\prime}(0)+B \varphi(0) \ni F \varphi, v(0)=$ $\varphi(0)$. As, in turn, $u_{\varphi}$ is a mild solution to $\dot{u}(t)+B u(t) \ni F(S(t) \varphi), u(0)=\varphi(0)$, we conclude from the usual integral inequality for mild solutions that, for $t>0$, $e^{-\omega t}\left\|u_{\varphi}(t)-v(t)\right\| \leq \int_{0}^{t} e^{-\omega \tau}\|F(S(\tau) \varphi)-F \varphi\| d \tau$. Thus,

$$
\begin{aligned}
& \left\|\frac{u \varphi(t)-u(0)}{t}-\frac{1}{t} \int_{0}^{t} w(\tau) d \tau\right\| \\
& =\left\|\frac{u \varphi(t)-\varphi(0)}{t}-\frac{v(t)-\varphi(0)}{t}\right\| \leq e^{\omega t} \frac{1}{t} \int_{0}^{t} e^{-\omega \tau}\|F(S(\tau) \varphi)-F \varphi\| d \tau .
\end{aligned}
$$

This shows that $u_{\varphi}^{\prime+}(0)$ exists and equals $w(0)=\varphi^{\prime}(0)$.

Step 2:

$$
\lim _{h \rightarrow 0^{+}} \frac{S(h) \varphi-\varphi}{h}=\varphi^{\prime} .
$$

Proof. We consider the auxiliary linear operator $\tilde{A}$ in $E$ :

$$
\left\{\begin{array}{l}
D(\tilde{A})=\left\{\rho \in E \mid \rho^{\prime} \in E, \rho^{\prime}(0)=0\right\}, \\
\tilde{A} \rho:=-\rho^{\prime}, \rho \in D(\tilde{A}) .
\end{array}\right.
$$

Actually, this is the solution operator in $E$ associated with (PFDE) for $\hat{X}=X, \hat{E}=$ $E$, and $B=F=0$. Thus, $\tilde{A}$ is a linear, densely defined m-accretive operator in $E$, generating the $C_{0}-\operatorname{semigroup}(\tilde{S}(t))_{t \geq 0}$, given by $\tilde{S}(t) \rho(s)=\rho(0)$ for $-t \leq s \leq 0$, and $\tilde{S}(t) \rho(s)=\rho(t+s)$ for $s \leq-t$. Also, $\lim _{h \rightarrow 0^{+}} h^{-1}(\tilde{S}(h) \rho-\rho)=-\tilde{A} \rho=\rho^{\prime}$ for all $\rho \in D(\tilde{A})$. As this applies in particular to $\psi:=\varphi-e_{1} \varphi^{\prime}(0)$, with $e_{1}$ denoting the function $e_{1}: I \rightarrow \mathbb{R}, e_{1}(s)=e^{s}$, in order to prove (4.6), it is enough to show that

$$
\lim _{h \rightarrow 0^{+}}\left(\left(\frac{S(h) \varphi-\varphi}{h}\right)-\left(\frac{\tilde{S}(h) \psi-\psi}{h}\right)\right)=e_{1} \varphi^{\prime}(0) .
$$

By direct calculation, for $h>0$, and, for the finite-delay case $I=[-R, 0], h<R$,

$$
(S(h) \varphi-\varphi)(s)-(\tilde{S}(h) \psi-\psi)(s)= \begin{cases}u \varphi(h+s)-\varphi(0)+\left(1-e^{s}\right) \varphi^{\prime}(0), & -h \leq s \leq 0, \\ \left(e^{h+s}-e^{s}\right) \varphi^{\prime}(0), & s \leq-h .\end{cases}
$$

Thus,

$$
\frac{1}{h}(S(h) \varphi-\varphi)-\frac{1}{h}(\tilde{S}(h) \psi-\psi)=\frac{1}{h}\left(\eta_{h}+\rho_{h}\right),
$$


with

$$
\eta_{h}(s)=\left\{\begin{array}{l}
u_{\varphi}(h+s)-\varphi(0)+\left(1-e^{h+s}\right) \varphi^{\prime}(0), \quad-h \leq s \leq 0, \\
0, \quad s \leq-h,
\end{array}\right.
$$

and $\rho_{h}(s)=\left(e^{h+s}-e^{s}\right) \varphi^{\prime}(0), s \in I$.

As for $\eta_{h}$, consider the function $x: I \cup \mathbb{R}^{+} \rightarrow X$, given by $x(s)=0$ for $s \in I$, and $x(t)=u_{\varphi}(t)-\varphi(0)+\left(1-e^{t}\right) \varphi^{\prime}(0)$ for $t \geq 0$. Then, $\eta_{h}=x_{h}$, and thus, as $x_{0}=0$, by (E3) (b),

$$
\frac{1}{h}\left\|\eta_{h}\right\|=\frac{1}{h}\left\|x_{h}\right\| \leq M_{1}(h) \frac{1}{h} \max _{0 \leq t \leq h}\left\|u_{\varphi}(t)-\varphi(0)+\left(1-e^{t}\right) \varphi^{\prime}(0)\right\| .
$$

Noting that $u \varphi(t)-\varphi(0)+\left(1-e^{t}\right) \varphi^{\prime}(0)=\left(u \varphi(t)-\varphi(0)-t \varphi^{\prime}(0)\right)-\left(\left(e^{t}-1\right)-t\right) \varphi^{\prime}(0)$, and recalling that $u_{\varphi}^{\prime+}(0)=\varphi^{\prime}(0)$ (Step 1 above), we conclude that

$$
\frac{1}{h}\left\|\eta_{h}\right\| \rightarrow 0 \quad \text { as } \quad h \rightarrow 0^{+} \text {. }
$$

As for $\rho_{h}$, we consider the function $\tilde{\rho}_{h}:=\frac{1}{h} \rho_{h}-e_{1} \varphi^{\prime}(0) \in E$. It solves the differential equation $\tilde{\rho}_{h}-\tilde{\rho}_{h}^{\prime}=0, \tilde{\rho}_{h}(0)=\frac{1}{h}\left(e^{h}-1\right) \varphi^{\prime}(0)-\varphi^{\prime}(0)$, so that, by (E2),

$$
\left\|\frac{1}{h} \rho_{h}-e_{1} \varphi^{\prime}(0)\right\| \leq\left|\frac{1}{h}\left(e^{h}-1\right)-1\right|\left\|\varphi^{\prime}(0)\right\| \rightarrow 0 \quad \text { as } \quad h \rightarrow 0^{+} .
$$

(4.8)-(4.10) imply (4.7), and thus complete the proof of (4.6).

Step 3:

$$
\frac{d}{d t} S(t) \varphi=U(t, 0) \varphi^{\prime} \quad \text { on } \quad(0, \infty) .
$$

Proof. In order to transfer differentiability of $S(\cdot) \varphi$ from $t=0$ to $t>0$, we invoke Lemma 4.7. Given $t>0$, and letting $\psi=S(h) \varphi$ for $h>0$ (with $h<R$ in the finite-delay case $I=[-R, 0]),(4.4)$ implies

$$
\begin{aligned}
& \|S(t+h) \varphi-S(t) \varphi-U(t, 0)(S(h) \varphi-\varphi)\| \\
& \quad \leq C_{1}(t) e^{C_{2}(t)} \int_{0}^{t}\|F(S(\tau+h) \varphi)-F(S(\tau) \varphi)-\tilde{F}[S(\tau) \varphi](S(\tau+h) \varphi-S(\tau) \varphi)\| d \tau .
\end{aligned}
$$

Claim: Given $\epsilon>0$, there exists $\delta>0$, such that

$$
\begin{aligned}
& \|F(S(\tau+h) \varphi)-F(S(\tau) \varphi)-\tilde{F}[S(\tau) \varphi](S(\tau+h) \varphi-S(\tau) \varphi)\| \\
& \leq \epsilon\|S(h) \varphi-\varphi\| \text { for all } h \in[0, \delta) \text { and all } \tau \in[0, t] .
\end{aligned}
$$

Proof. Here, we use the "convexity-trick" of [69, Step 2 of the proof of Thm. 2.3] (or of [42, Lemma II.4.3] for "regular" differentiability on open sets) which, for the sake of completeness, we briefly reproduce here: Given a mapping $H: D(H) \subset$ $X \rightarrow Y, X$ and $Y$ real Banach spaces, that is continuously F-differentiable on a convex subset $C \subset D(H)$ in the sense of Definiton 4.1, part 2, and given $x, y \in C$, define the map $R:[0,1] \rightarrow Y$ by

$$
R(\alpha):=H(x+\alpha(y-x))-\alpha \tilde{H}[x](y-x) .
$$


Then, $R^{\prime}(\alpha)=(\tilde{H}[x+\alpha(y-x)]-\tilde{H}[x])(y-x)$ for all $0<\alpha<1$. By continuity of both $R$ and the differential map,

$$
R(1)-R(0)=\int_{0}^{1}(\tilde{H}[x+\alpha(y-x)]-\tilde{H}[x])(y-x) d \alpha .
$$

Specializing this result to $H:=\left.F\right|_{\hat{E}}$, and letting $C:=C(\varphi)$ be the closed convex hull of the set $\{S(s) \varphi \mid s \in[0, t+1]\}$ (and recalling that, in Theorem 4.5, we have assumed $\hat{E}$ to be (closed and) convex), we consider, for $h \in[0,1)$, and $\tau \in[0, t]$,

$R(\alpha):=F(S(\tau) \varphi+\alpha(S(\tau+h) \varphi-S(\tau) \varphi))-\alpha \tilde{F}[S(\tau) \varphi](S(\tau+h) \varphi-S(\tau) \varphi), \alpha \in[0,1]$, and read from (4.14) that

$$
\begin{aligned}
& \|F(S(\tau+h) \varphi)-F(S(\tau) \varphi)-\tilde{F}[S(\tau) \varphi](S(\tau+h) \varphi-S(\tau) \varphi)\| \\
& \quad \leq\|S(\tau+h) \varphi-S(\tau) \varphi\| \int_{0}^{1}\|\tilde{F}[S(\tau) \varphi+\alpha(S(\tau+h) \varphi-S(\tau) \varphi)]-\tilde{F}[S(\tau) \varphi]\| d \alpha .
\end{aligned}
$$

The set $C(\varphi)$ is (convex and) compact; hence, the (continuous) differential map $\{\psi \rightarrow \tilde{F}[\psi]\}$ is uniformly continuous on $C(\varphi)$. Let $\epsilon>0$, and choose $0<\delta_{1}<1$ such that, if $\psi, \rho \in C(\varphi)$, with $\|\psi-\rho\|<\delta_{1}$, then $\|\tilde{F}[\psi]-\tilde{F}[\rho]\|<\epsilon e^{-\gamma t}$ (with $\gamma$ as in Theorem 2.1 (c)). Then, choose $0<\delta_{2}<1$ such that, for $h \in\left[0, \delta_{2}\right),\|S(h) \varphi-\varphi\|<$ $\delta_{1} e^{-\gamma t}$. Then,

$$
\begin{gathered}
(S(\tau) \varphi+\alpha(S(\tau+h) \varphi-S(\tau) \varphi)), S(\tau) \varphi \in C(\varphi), \text { and } \\
\|(S(\tau) \varphi+\alpha(S(\tau+h) \varphi-S(\tau) \varphi)-S(\tau) \varphi)\|=\alpha\|S(\tau+h) \varphi-S(\tau) \varphi\|<\delta_{1},
\end{gathered}
$$

and thus

$$
\|\tilde{F}[S(\tau) \varphi+\alpha(S(\tau+h) \varphi-S(\tau) \varphi)]-\tilde{F}[S(\tau) \varphi]\|<\epsilon e^{-\gamma t}
$$

for all $0 \leq h \leq \delta_{2}$, and all $\alpha \in[0,1]$, and $\tau \in[0, t]$. By (4.15), this proves (4.13).

(4.12) combined with (4.13) implies that, for any $t>0$, given any $\epsilon>0$, there exists $\delta>0$ such that

$$
\|S(t+h) \varphi-S(t) \varphi-U(t, 0)(S(h) \varphi-\varphi)\|<\epsilon\|S(h) \varphi-\varphi\| \quad \text { for all } h \in[0, \delta) .
$$

In combination with (4.6) from Step 2 above, and recalling that the map $U(t, 0)$ is linear, this, at first, implies (4.11) for differentiability from the right. But, as $U(t, s), 0 \leq s \leq t$, is a strongly continuous evolution system, (4.17) serves to prove (4.11) altogether.

Interlude: So far, (4.11), in conjunction with Lemma 4.7 (a), serves to prove parts (ii) and (iii) of Theorem 4.5, as well as, in conjunction with (E1) (a), continuous differentiability of $u_{\varphi}$.

In the previously known cases without flow-invariance, as reported in Remark 4.6, part 1, above, and even under the local range conditions for flow-invariance as in 67, Thm. 2.1], (4.11) would also suffice to prove the classical solution part of Theorem 4.5(i): in any of these cases, we have the range condition $R(I+\lambda A) \supset \operatorname{cl} D(A), 0<\lambda$ small enough, for the associated solution operator $A$ in $E$ (which is closed according to (E1) (d)), and it is a general fact of nonlinear evolution equations that under this condition, if, for $\left(A\right.$ closed and) $\varphi \in c l D(A), \dot{S}\left(t_{0}\right) \varphi:=\left[\frac{d}{d t} S(t) \varphi\right]_{t=t_{0}}$ exists for some $t_{0}>0$, then $S\left(t_{0}\right) \varphi \in D(A)$, and $\dot{S}\left(t_{0}\right) \varphi+A S\left(t_{0}\right) \varphi \ni 0$; cf. 48, Thm. 
4.5, p. 81]. It is unclear to the author whether this result also holds under the weaker subtangential condition $H(0,0)$ of Step 2 of the proof of Theorem 2.1 in our context.

Notice, however, that we need the classical-solution part only on the function $u_{\varphi}(\cdot)=(S(\cdot) \varphi)(0)$, and not on the motion $S(\cdot) \varphi$.

Step 4: $u_{\varphi}$ is a classical solution to (PFDE).

So far, we know from (4.11), in conjunction with (E1) (a), that $\dot{u}_{\varphi}(t)$ exists for all $t>0$. By general regularity theory ([7, Cor. 7.5]) for $B \omega-\mathrm{m}$-accretive, we conclude that, for $t>0, u_{\varphi}(t) \in D(B)$, and $\dot{u}_{\varphi}(t)+B u_{\varphi}(t) \ni F\left(\left(u_{\varphi}\right)_{t}\right)$. As, by Step 1 above, $u_{\varphi}^{\prime+}(0)=\varphi^{\prime}(0)$, and $\varphi \in D(A)$, and thus $\varphi^{\prime}(0)+B \varphi(0) \ni F(\varphi)$, this holds as well for $t=0$. Hence, $u_{\varphi}$ is a classical solution to (PFDE), and the proof of Theorem 4.5 is complete.

\section{Population models}

As indicated in the Introduction, the existence results of Section 2 are particularly convenient for applications to population models in the $L^{1}$-context. We exemplify this in this section with a class of prominent models.

5.1. Conventions for this section:

1. $\Omega$ is an open subset of $\mathbb{R}^{N}$.

2. Given $\beta \geq 0,[0, \beta]$ denotes the order-interval of all $x \in L^{1}(\Omega)$ such that $0 \leq x(\omega) \leq \beta$ a.e. $\omega \in \Omega$.

3. For a function $u: \mathbb{R}^{+} \rightarrow X, X$ a Banach space, $\omega(u):=\left\{x \in X \mid u\left(t_{n}\right) \rightarrow x\right.$ for some sequence $\left.t_{n} \rightarrow \infty\right\}$, respectively $\omega_{w}(u):=\left\{x \in X \mid u\left(t_{n}\right) \rightarrow x\right.$ weakly for some sequence $\left.t_{n} \rightarrow \infty\right\}$, denotes the (norm) omega-limit set, respectively the weak omega-limit set of $u$.

The following is a prototype model for a spatially distributed population with delay in the birth process:

$$
\left\{\begin{array}{l}
\frac{\partial}{\partial t} u(x, t)-d \Delta u(x, t)=a u(x, t)\left[1-b u(x, t)-\int_{-1}^{0} u(x, t+r(s)) d \eta(s)\right] \\
u_{\mid[-R, 0]}=\varphi \in C([-R, 0], X) \\
+ \text { boundary conditions, }
\end{array}\right.
$$

where $a, b, d>0, \eta$ is a positive bounded regular Borel measure on $[-1,0]$ with $(\|\eta\|>0$ and $) b+\|\eta\|=1$, and $r:[-1,0] \rightarrow[-R, 0]$ is a continuous delay function, and $X$ is an appropriate state space of real-valued functions on $\Omega$. In particular, this equation serves as a model for the density of red blood cells in an animal. This model (and related ones with slightly different or more general history-responsive functions) has been considered by a number of authors under both Dirichlet ( 52 , 53]) and (linear) Neumann boundary conditions ([29, 43, 44, 45, 46, 47, 49, 74, 88, ). Because of the quadratic terms arising in the history-responsive function $F(\varphi)=$ $a \varphi(0)\left[1-b \varphi(0)-\int_{-1}^{0} \varphi(r(s)) d \eta(s)\right]$, and thus the problem of restricting existence results to function spaces on $\Omega$ invariant under products, in these references the state spaces have been restricted to $\Omega$ bounded, and either spaces of functions continuous up to the closure of $\Omega([43,44,45,49,52,53,74])$, or to "small" Sobolev spaces and corresponding restricted initial histories such as $\varphi \in C\left([-R, 0], W^{2, p}(\Omega)\right)$ 
with $p>N([29,46,47,88])$. However, with $u(\cdot, t)$ representing a population density and thus the $L^{1}$-norm of $u(\cdot, t)$ being a measure of the total population at time $t$, the natural state space obviously is $L^{1}(\Omega)$.

The flow-invariance results of Section 2 can now aptly be applied to derive existence results in this natural state space for this and related models.

At first, though, we wish to extend the model to more general diffusion operators. Notice that, in the model (5.1), the Laplacian models uniform diffusion of the population all throughout the habitat. However, in practice, diffusion may depend (even nonlinearly) on the place in space, and, too, there may be sources and/or sinks. Thus, we wish to place our models in the broader setting of general nonlinear diffusion/absorption processes associated with the differential expression

$$
-\operatorname{div} a(\cdot, \operatorname{grad} u)+\tilde{\beta}(u)+\text { boundary conditions. }
$$

Assumptions 83, 84, 85, 86]

(1) The vector field $a: \Omega \times \mathbb{R}^{N} \rightarrow \mathbb{R}^{N}$ satisfies the following conditions:

(H1) $a: \Omega \times \mathbb{R}^{N} \rightarrow \mathbb{R}^{N}$ is a Carathéodory function, i.e., measurable in $x \in \Omega$ for all $\xi \in \mathbb{R}^{N}$, and continuous in $\xi \in \mathbb{R}^{N}$ for a.e. $x \in \Omega$; $a(\cdot, 0)=0$;

(H2) "monotonicity condition": $(a(x, \xi)-a(x, \hat{\xi})) \cdot(\xi-\hat{\xi}) \geq 0$ for all $\xi, \hat{\xi} \in \mathbb{R}^{N}$, a.e. $x \in \Omega$

(H3) "coerciveness condition": $a(x, \xi) \cdot \xi \geq \lambda_{0}|\xi|^{p}-a_{0}(x)$ for all $\xi \in$ $\mathbb{R}^{N},|\xi| \geq R_{0}$, a.e. $x \in \Omega$, where $1<p<\infty, a_{0} \in L^{1}(\Omega), \lambda_{0}>$ $0, R_{0} \geq 0$

(H4) $|a(x, \xi)| \leq b_{0}(x)+C_{0}|\xi|^{p-1}$ for all $\xi \in \mathbb{R}^{N}$, a.e. $x \in \Omega$, where $b_{0} \in L^{p^{\prime}}(\Omega)+L^{\infty}(\Omega), 1 / p+1 / p^{\prime}=1, C_{0} \geq 0$.

(2) $j: \Omega \times \mathbb{R} \rightarrow[0, \infty]$ is a function satisfying

(a) $j(\cdot, r)$ is measurable for any $r \in \mathbb{R}$,

(b) $j(x, \cdot)$ is convex, lower semicontinuous with $j(x, 0)=0$ for a.e. $x \in \Omega$, and $\beta(x, r):=\partial j(x, \cdot)(r)$ for $r \in \mathbb{R}$, a.e. $x \in \Omega$, i.e., $\beta(x, \cdot)$ is the subdifferential of the convex function $j(x, \cdot)$, which is defined as usual by $t \in \partial j(x, r) \Leftrightarrow j(x, s) \geq j(x, r)+t(s-r)$ for all $s \in \mathbb{R}$.

Defining the operator $A_{0}$ in $L^{1 \cap \infty}(\Omega)$ by

$$
\left\{\begin{array}{c}
D\left(A_{0}\right)=\left\{u \in L^{1 \cap \infty}(\Omega) \mid \xi \cdot u \in W_{0}^{1, p}(\Omega) \text { for all } \xi \in C_{0}^{\infty}\left(\mathbb{R}^{N}\right),\right. \\
\left.|a(\cdot, \operatorname{grad} u)| \in L^{p^{\prime}+\infty}(\Omega),-\operatorname{div} a(\cdot, \operatorname{grad} u) \in L^{1 \cap \infty}(\Omega)\right\}, \\
A_{0} u:=-\operatorname{div} a(\cdot, \operatorname{grad} u) \text { in the sense of distributions, } u \in D\left(A_{0}\right),
\end{array}\right.
$$

let $A:={\overline{A_{0}}}^{L^{1}(\Omega)}$ be the closure of $A_{0}$ in $L^{1}(\Omega)$. With the absorption operator $C \subset$ $L^{1}(\Omega) \times L^{1}(\Omega)$ defined by $w \in C u \Leftrightarrow u, w \in L^{1}(\Omega), w(x) \in \partial j(x, u(x)) \quad \mu$-a.e. $x \in \Omega$, the operator $B:=A+C$ is a realization of the diffusion/absorption operator with Dirichlet boundary conditions of (5.2) above in $L^{1}(\Omega)$. The operator $B=A+C$ just defined is m-completely accretive in case $j$ does not depend on the space variable, or if $j(\cdot, r) \in L^{1+\infty}(\Omega)$ in the general case. (For more general conditions to this effect, cf. [8.) Corresponding statements hold for diffusion operators $A$ as above with (generally nonlinear) Neumann boundary conditions such as

$$
-\operatorname{div} a(\cdot, \operatorname{grad} u) ; \quad-a(\cdot, \operatorname{grad} u) \cdot n \in \beta(u) \quad \text { on } \quad \partial \Omega
$$

with $\beta \subset \mathbb{R}^{2}$ a maximal monotone graph with $0 \in \beta(0)$, for they can also be realized by $\mathrm{m}$-completely accretive operators in $L^{1}(\Omega)$ (cf. [4, 83, 84, 85, 86]). 
In order to achieve the desired order of generality, we consider the model (5.1) with the Laplacian being replaced by any m-completely accretive operator $B \subset$ $L^{1}(\Omega) \times L^{1}(\Omega):$

$$
\left\{\begin{array}{l}
\dot{u}(t)+B u(t) \ni a u(t)\left[1-b u(t)-\int_{-1}^{0} u(t+r(s)) d \eta(s)\right], \quad t \geq 0 \\
u_{\mid[-R, 0]}=\varphi
\end{array}\right.
$$

Proposition 5.1. For the delay equation (5.4), with $B \subset L^{1}(\Omega) \times L^{1}(\Omega) m$ completely accretive with $(0,0) \in B$, with initial-history space $E:=C\left([-R, 0], L^{1}(\Omega)\right)$, and with $\beta_{0}:=1 / b$, the following hold:

(a) For all $\varphi \in E$ with $\varphi(0) \in \operatorname{cl} D(B) \cap L^{\infty}(\Omega)$, and $\varphi(s)(\omega) \geq 0$ a.e. $\omega \in \Omega$ for all $s \in[-R, 0]$, there exists a unique global mild solution $u_{\varphi}$ to (5.4) with $u_{\varphi}(t) \in\left[0, \max \left\{\beta_{0},\|\varphi(0)\|_{\infty}\right\}\right]$ for all $t \geq 0$. If, in addition, $\varphi(s) \in[0, \beta]$ for all $s \in[-R, 0]$, for some $\beta \geq \beta_{0}$, then $\left(u_{\varphi}\right)_{t} \in[0, \beta]$ for all $t \geq 0$.

(b) If, for $\varphi$ as in (a), and for some $\beta \geq \beta_{0}, u_{\varphi}\left(t_{0}\right) \in[0, \beta]$ for some $t_{0} \geq 0$, then $u_{\varphi}(t) \in[0, \beta]$ for all $t \geq t_{0}$.

(c) For all $\varphi$ as in (a),

$$
\lim _{t \rightarrow \infty} \operatorname{dist}\left(u_{\varphi}(t),\left[0, \beta_{0}\right]\right)=0
$$

(c1) If $u_{\varphi}$ has relatively compact range, then $\omega\left(u_{\varphi}\right) \subset\left[0, \beta_{0}\right]$.

(c2) $u_{\varphi}$ has relatively compact range if $\Omega$ has finite Lebesgue measure, and either (i) $u_{\varphi}$ is uniformly continuous and $J_{\lambda}^{B}$ transforms $L^{\infty}$-bounded subsets of $L^{1}(\Omega)$ into $L^{1}(\Omega)$-relatively compact sets for $\lambda>0$ small enough, or (ii) the semigroup $\left(S_{B}(t)\right)_{t \geq 0}$ generated by $-B$ transforms $L^{\infty}$-bounded subsets of $L^{1}(\Omega)$ into $L^{1}(\Omega)$-relatively compact sets.

(c3) In case $\Omega$ has finite Lebesgue-measure, $u_{\varphi}$ has weakly relatively compact range, and $\omega_{w}\left(u_{\varphi}\right) \subset\left[0, \beta_{0}\right]$.

(d) For all $\varphi$ as in (a),

$$
\left\|u_{\varphi}(t)\right\| \leq e^{a t}\|\varphi(0)\| \quad \text { for all } t \geq 0
$$

and if, in addition, $(B+\omega I)$ is accretive for some $\omega<0$, then

$$
\left\|u_{\varphi}(t)\right\| \leq e^{(\omega+a) t}\|\varphi(0)\| \quad \text { for all } t \geq 0 .
$$

In particular, if $\omega<0$, and $a<-\omega$, then all these solutions tend exponentially to the zero-function.

As the proof of Proposition 5.1 carries over almost verbatim (except for the constants) to further population models, we include one more concrete model, and consider the following variant of model (5.4):

$$
\left\{\begin{array}{l}
\dot{u}(t)+B u(t) \ni u(t)\left[1+a u(t)-b(u(t))^{2}\right. \\
\left.\quad-(1+a-b) \int_{-R}^{0} f(s) u(t+s) d s\right], t \geq 0 \\
u_{\mid[-R, 0]}=\varphi
\end{array}\right.
$$

for $\Omega$ open in $\mathbb{R}^{N}, B \subset L^{1}(\Omega) \times L^{1}(\Omega)$ m-completely accretive (such as (5.2) above), $a, b>0, b<1+a$, and $f \in L^{1}((-R, 0))$ nonnegative with $\|f\|_{1}=1$. Once again, for $\Omega$ bounded, $B=-\Delta$ with $0-$ Neumann boundary conditions, and state space $C(\bar{\Omega})$; see [27, 61 . 
Proposition 5.2. For the delay equation (5.6), with $B \subset L^{1}(\Omega) \times L^{1}(\Omega) m$ completely accretive with $(0,0) \in B$, and with initial-history space $E:=C([-R, 0]$, $L^{1}(\Omega)$ ), all of the assertions of Proposition 5.1 hold, with the lower bound $\beta_{0}$ for the upper bound of flow-invariant order intervals $[0, \beta]$ here given by $\beta_{0}=$ $(2 b)^{-1}\left(a+\sqrt{a^{2}+4 b}\right)$, and the constant a in proposition (d) being replaced by $\left(1+\frac{a^{2}}{4 b}\right)$.

Remarks 5.3. 1. The main point of Propositions 5.1 and 5.2 clearly is the existence and flow-invariance result of (a). It seems to be a particular advantage of the results of section 2 that, for population models such as (5.4) and (5.6), these results can now be achieved for the natural state space $L^{1}$ (and without a boundedness requirement on $\Omega$ ), rather than for either $C(\bar{\Omega})$ or $W^{2, p}(\Omega)$ (with $\Omega$ bounded) as previously (see the discussion on model (5.1) above). It is particularly satisfying that this works under the minimal hypotheses that the initial history be nonnegative throughout the history, and, at $t=0, \varphi(0)$ be $L^{\infty}$-bounded, as these, too, are natural assumptions on population densities.

2. Also, the fact that the upper bound $\beta$ of those order intervals $[0, \beta]$ that are flowinvariant is bounded below by $\beta_{0}=1 / b$, respectively, $\beta_{0}=(2 b)^{-1}\left(a+\sqrt{a^{2}+4 b}\right)$, comes as no surprise: even for the very special 1-dimensional case of model (5.1) for $\Omega=(0, \pi)$, and Dirichlet boundary conditions, it is known [72, Thm. 4.9, (2)] that, for $a>d$, the zero-equilibrium is not $L^{\infty}$-stable; thus, $\beta>0$ cannot be arbitrarily small.

Further, with regard to (c), the attracting order-interval $\left[0, \beta_{0}\right]$ seems to be best possible: for model (5.4), by our normalizations of $b,\|\eta\|>0$, and $b+\|\eta\|=1$, $\beta_{0}=1 / b$ can be arbitrarily close to 1 , while, say, for the particular model (5.1) with ( $\Omega$ bounded, and) Neumann boundary conditions, the constant $\mathbf{1}$-function is an equilibrium solution.

3. With the generality both in the operator $B$ and the boundary conditions (such as (5.3), for instance), the asymptotic and stability results of (c) and (d) can only serve as first approximations. More detailed analyses on the asymptotic behaviour of solutions, in particular, special equilibria and/or convergence to such, may start from here, but, obviously, now depend on particular choices of $\Omega, B$, and the boundary conditions.

3.1. Both of the special assumptions of (c2) hold, for instance, for ( $\Omega$ bounded, and) $B=-\Delta$, or even some of the nonlinear analogues $B$ as in (5.2), with Dirichlet boundary conditions; cf. [78, Ch. 2.6] and [83, 84, 86].

3.2. A further general tool for the asymptotic analysis of solutions to models such as (5.4) and (5.6) is the comparison principle for completely accretive operators: if $B \subset L^{1}(\Omega) \times L^{1}(\Omega)$ is m-completely accretive, and if $u$, respectively $v$, is a mild solution to $\dot{u}(t)+B u(t) \ni f(t)$, respectively $\dot{v}(t)+B v(t) \ni g(t), t \geq 0$, then ([7, Thm. 19.12])

$$
\begin{array}{r}
\left\|(u(t)-v(t))^{+}\right\| \leq\left\|(u(s)-v(s))^{+}\right\|+\int_{s}^{t}[u(\tau)-v(\tau), f(\tau)-g(\tau)]_{+} d \tau \\
\quad \text { for all } 0 \leq s \leq t,
\end{array}
$$

where, for $x, y \in L^{1}(\Omega),[x, y]_{+}:=\partial N(x, y)$ for $N(x):=\left\|x^{+}\right\|$. For instance, in the context of model (5.4), one can show that for any given $\varphi$ as in Proposition 5.1 (a), $0 \leq u_{\varphi}(t) \leq v(t)$ for all $t \geq 0$, where $v$ is the mild solution to $\dot{v}(t)+B v(t) \ni$ $a v(t), t \geq 0, v(0)=\varphi(0)$, by using this "order"-integral inequality in conjunction 
with the particular result of Proposition 5.1 (a) that $u_{\varphi}(t)$ is non-negative for all $t \geq 0$.

4. While the history-responsive operators $F$ of the above models are not differentiable (in the sense of Definition 4.1) in $L^{1}(\Omega)$, there is an enormous recent activity on regularity properties of mild solutions to the inhomogeneous Cauchy problem for operators $B$ in $L^{1}$ as in (5.2) (well-known, anyway, for $B=-\Delta$ ). For results on mild solutions being distributional, or entropy, or renormalized solutions, the reader is referred to $3,4,5,73$ and further references therein.

Proof of Proposition 5.1. Step 1: We start with a technical result on the asymptotic behaviour of mild solutions to the inhomogeneous Cauchy problem.

Notation: 1. Given a convex function $N: X \rightarrow \mathbb{R}, X$ a Banach space, we let

$$
\partial N(x, y):=\lim _{\lambda \rightarrow 0^{+}} \frac{N(x+\lambda y)-N(x)}{\lambda}=\inf _{\lambda>0} \frac{N(x+\lambda y)-N(x)}{\lambda}, \quad x, y \in X .
$$

2. For $x \in L^{1}(\Omega)$, and $r \in \mathbb{R},(x=r):=\{\omega \in \Omega \mid x(\omega)=r\}$.

Lemma 5.4. Let $B \subset X \times X$ be an $\omega$-accretive operator in a Banach space $X, C \subset X$ a closed convex subset of $X$, and let $N(x):=\operatorname{dist}(x, C), x \in X$. Moreover, assume that, for $\lambda>0$ small enough, $R(I+\lambda B) \supset C$, and $J_{\lambda}{ }^{B} C \subset C$. Then we have:

(a) $N$ is convex and continuous, and $\partial N(x, y) \geq-\omega N(x)$ for all $(x, y) \in B$.

(b) If, in addition, $B$ is $\omega$-m-accretive, then, for all $f \in L_{l o c}^{1}\left(\mathbb{R}^{+}, X\right)$, and $u$ : $\mathbb{R}^{+} \rightarrow X$ a mild solution to $\dot{u}(t)+B u(t) \ni f(t), t \geq 0$,

$$
N(u(t)) \leq N(u(s))+\int_{s}^{t}(\partial N(u(\tau), f(\tau))+\omega N(u(\tau))) d \tau \quad \text { for all } 0 \leq s \leq t .
$$

(c) If, in the context of (b), $\partial N(u(t), f(t)) \leq-\omega N(u(t))$ for any $t \geq 0$ for which $N(u(t))>0$, then we have: if $u\left(t_{0}\right) \in C$ for some $t_{0} \geq 0$, then $u(t) \in C$ for all $t \geq t_{0}$.

(Proposition (b) of Lemma 5.4 is the result of [22, Lemma 2].)

Proof of Lemma 5.4. (a): The only nontrivial assertion is the inequality: let $(x, y) \in$ $B, \lambda>0$ small enough, and let $\left(c_{n}\right)_{n} \subset C$ such that $\left\|(x+\lambda y)-c_{n}\right\|$ $\rightarrow \operatorname{dist}(x+\lambda y, C)=N(x+\lambda y)$. Then $N(x)=\operatorname{dist}(x, C) \leq\left\|x-J_{\lambda}{ }^{B} c_{n}\right\|=$ $\left\|J_{\lambda}{ }^{B}(x+\lambda y)-J_{\lambda}{ }^{B} c_{n}\right\| \leq(1-\lambda \omega)^{-1}\left\|(x+\lambda y)-c_{n}\right\| \rightarrow(1-\lambda \omega)^{-1} N(x+\lambda y)$. Rearranging yields $(1 / \lambda)(N(x+\lambda y)-N(x)) \geq-\omega N(x)$ for all $\lambda>0$ small enough.

Proposition (b) now also follows from (a) and [7, Thm. 19.8].

As for (c), assume that $u\left(t_{0}\right) \in C$ for some $t_{0} \geq 0$, but $u\left(t_{1}\right) \notin C$ for some $t_{1}>t_{0}$. Let $t_{2}:=\inf \left\{t_{0}<t \leq t_{1} \mid u(s) \notin C\right.$ for all $\left.s \in\left[t, t_{1}\right]\right\}$. Then $u\left(t_{2}\right) \in C$ (clear in case $t_{2}=t_{0}$, and a consequence of continuity of both $N$ and $u$ in case $\left.t_{2}>t_{0}\right)$, and $u(t) \notin$ $C$, and thus $N(u(t))>0$ for all $t \in\left(t_{2}, t_{1}\right]$. However, from (b) and the particular assumption of (c), $N(u(t)) \leq N\left(u\left(t_{2}\right)\right)+\int_{t_{2}}^{t}(\partial N(u(\tau), f(\tau))+\omega N(u(\tau))) d \tau \leq 0$ for all $t \in\left(t_{2}, t_{1}\right]$. This contradiction completes the proof of Lemma 5.4.

Proof of Proposition 5.1. (a): Here, we turn to Corollary 2.6: With $E=C([-R, 0]$, $\left.L^{1}(\Omega)\right)$ and, for $\beta>0, \hat{X}:=\hat{X}_{\beta}=[0, \beta]$, we let $\hat{E}_{0}=\hat{E}_{0, \beta}=\{\varphi \in E \mid \varphi(0) \in$ cl $\left.D(B) \cap \hat{X}_{\beta}\right\}$ (with reference to Remark 2.2, part 1), and $\hat{E}=\hat{E}_{\beta}^{+}:=\left\{\varphi \in \hat{E}_{0, \beta} \mid\right.$ $\varphi(s) \geq 0$ for all $s \in[-R, 0]\}$. Also, with $G: E \rightarrow L^{1}(\Omega), G(\varphi):=\int_{-1}^{0} \varphi(r(s)) d \eta(s)$, 
we let, $F: \hat{E}_{0, \beta} \rightarrow L^{1}(\Omega), F(\varphi):=a \varphi(0)[1-b \varphi(0)-G \varphi]$, and, momentarily, $F^{+}$: $\hat{E}_{0, \beta} \rightarrow L^{1}(\Omega), F^{+}(\varphi):=a \varphi(0)\left[1-b \varphi(0)-(G \varphi)^{+}\right]$. Then, for $B, \hat{X}_{\beta}, \hat{E}_{0, \beta}, \hat{E}_{\beta}^{+}$, and $F^{+}$, all of assumptions (A1)-(A4) are fulfilled, with $L_{F^{+}}=a \beta|\eta|$, and $M_{F^{+}}=$ $a(1+\beta|\eta|)$. Here, the only nontrivial part is (A4), (c), and this even holds for all $\varphi, \psi \in \hat{E}_{0, \beta}$ : let $\alpha \in J(\varphi(0)-\psi(0))$, i.e., $\alpha \in L^{\infty}(\Omega),|\alpha| \leq 1$, and $\alpha(\varphi(0)-$ $\psi(0))=|\varphi(0)-\psi(0)|$ a.e. on $\Omega$. Hence, both of $\int \alpha(\varphi(0)-\psi(0))(\varphi(0)+\psi(0))$ and $\int \alpha(\varphi(0)-\psi(0))(G \varphi)^{+}$are nonnegative, and thus

$$
\begin{aligned}
& \left\langle\alpha, F^{+} \varphi-F^{+} \psi\right\rangle=a \int \alpha(\varphi(0)-\psi(0)) \\
& -a b \int \alpha(\varphi(0)-\psi(0))(\varphi(0)+\psi(0)) \\
& -a \int \alpha(\varphi(0)-\psi(0))(G \varphi)^{+}-a \int \alpha \psi(0)\left((G \varphi)^{+}-(G \psi)^{+}\right) \\
& \leq a(1+\beta|\eta|)\|\varphi-\psi\| .
\end{aligned}
$$

We next show that the subtangential condition (STC) $)_{X}$ of Corollary 2.6 is fulfilled for any $\beta \geq 1 / b$, and (even) for all $\varphi \in \hat{E}_{0, \beta}$. We have to show that, given $\varphi \in$ $\hat{E}_{0, \beta}$, (i) $F^{+}(\varphi) \geq 0$ on $\left(\varphi(0)=0\right.$ ), and (ii) $F^{+}(\varphi) \leq 0$ on $(\varphi(0)=\beta)$. However, on $(\varphi(0)=0), F^{+}(\varphi)=0$, and on $(\varphi(0)=\beta), F^{+}(\varphi)=a \beta\left(1-b \beta-(G \varphi)^{+}\right) \leq 0$, since $\beta \geq 1 / b$. Thus, according to Corollary 2.6 , for any $\beta \geq(1 / b)$, given any $\varphi \in \hat{E}=$ $\hat{E}_{\beta}^{+}$, there exists a global mild solution $u_{\varphi}$ to $\dot{u}(t)+B u(t) \ni F^{+}\left(u_{t}\right), u_{\mid[-R, 0]}=\varphi$ with $\left(u_{\varphi}\right)_{t} \in \hat{E}_{\beta}^{+}$for all $t \geq 0$. However, on $\hat{E}_{\beta}^{+}, F^{+}=F$, so that, in fact, $u_{\varphi}$ is a mild solution to (5.4). Uniqueness now follows from Theorem 2.1, (b), in conjunction with the fact that, by $(5.8),[\varphi(0)-\psi(0), F \varphi-F \psi] \leq a(1+\beta|\eta|)\|\varphi-\psi\|$ for all $\varphi, \psi \in \hat{E}_{\beta}^{+}$. These arguments serve to prove the first part of assertion (a) of Proposition 5.1. The second part follows by replacing $\hat{E}=\hat{E}_{\beta}^{+}$by $\hat{E}=\left\{\varphi \in \hat{E}_{0, \beta} \mid\right.$ $\varphi(s) \in[0, \beta]$ for all $s \in[-R, 0]\}$.

Proof of Proposition 5.1. (b): Let $\beta \geq 1 / b$, let $\varphi \in E$ with $\varphi(0) \in \operatorname{cl} D(B) \cap$ $L^{\infty}(\Omega)$, and $\varphi(s) \geq 0$ for all $s \in[-R, 0]$, and let $u_{\varphi}$ be the unique mild solution to (5.4) as in proposition (a). According to Lemma 5.4, (c), we have to show that $\partial N_{\beta}\left(u_{\varphi}(t), F\left((u \varphi)_{t}\right) \leq 0\right.$ for any $t \geq 0$ for which $N_{\beta}\left(u_{\varphi}(t)\right)>0$, with $N_{\beta}(x)=$ $\operatorname{dist}(x,[0, \beta])$. It is not hard to see that, for $x, y \in L^{1}(\Omega)$,

$$
\partial N_{\beta}(x, y)=\int_{(x=0)} y^{-}+\int_{(x=\beta)} y^{+}-\int_{(x<0)} y+\int_{(x>\beta)} y .
$$

With $t \geq 0$, and $x=u_{\varphi}(t), y=F\left(\left(u_{\varphi}\right)_{t}\right)$, the first and the third term on the right vanish, and on $\left(u_{\varphi}(t) \geq \beta\right), F\left(\left(u_{\varphi}\right)_{t}\right) \leq 0$ (notice $\beta \geq 1 / b$, and $\left(u_{\varphi}\right)_{t} \geq 0$ on $[-R, 0])$. This implies the desired inequality.

Proof of Proposition 5.1. (c): From what we have just shown, in conjunction with (5.7), we already know that, for $\varphi$ as in (c), $\operatorname{dist}\left(u_{\varphi}(t),\left[0, \beta_{0}\right]\right) \searrow \delta \geq 0$ as $t \rightarrow \infty$. From (5.9) we read that

$$
\partial N_{\beta_{0}}\left(u \varphi(t), F\left((u \varphi)_{t}\right)\right)=\int_{\left(u \varphi(t)>\beta_{0}\right)} F\left((u \varphi)_{t}\right)
$$


Thus, invoking (5.7), for all $0 \leq s \leq t$,

$$
\begin{aligned}
0 & \leq a(t-s) \delta \leq a \int_{s}^{t} \operatorname{dist}\left(u_{\varphi}(\tau),\left[0, \beta_{0}\right]\right) d \tau \\
& =a \int_{s}^{t}\left(\int_{\left(u \varphi(\tau)>\beta_{0}\right)}\left(u_{\varphi}(\tau)-\beta_{0}\right)\right) d \tau=a \int_{s}^{t}\left(\int_{\left(u \varphi(\tau)>\beta_{0}\right)} 1 / b\left[b u_{\varphi}(\tau)-1\right]\right) d \tau \\
& \leq a \int_{s}^{t}\left(\int_{\left(u \varphi(\tau)>\beta_{0}\right)} u_{\varphi}(\tau)\left[b u_{\varphi}(\tau)+G\left(\left(u_{\varphi}\right)_{\tau}\right)-1\right]\right) d \tau \\
& =-\int_{s}^{t} \partial N_{\beta_{0}}\left(u_{\varphi}(\tau), F\left(\left(u_{\varphi}\right)_{\tau}\right)\right) d \tau \leq \operatorname{dist}\left(u_{\varphi}(s),\left[0, \beta_{0}\right]\right)-\operatorname{dist}\left(u_{\varphi}(t),\left[0, \beta_{0}\right]\right) \\
& \leq \operatorname{dist}\left(u_{\varphi}(s),\left[0, \beta_{0}\right]\right) .
\end{aligned}
$$

This shows that $\delta=0$, and proves the claim of (c).

With, at this point, (c1) being obvious, we turn to (c2). As for (c2), (i), we need the following technical result, which is a direct consequence of the integral inequality for mild solutions:

Lemma 5.5 ([7, Lemma 14.7]). If $C \subset X \times X$ is $\omega$-accretive, $f \in L_{l o c}^{1}\left(\mathbb{R}^{+}, X\right)$, and $u: \mathbb{R}^{+} \rightarrow X$ is a mild solution to $\dot{u}+C u \ni f$ on $\mathbb{R}^{+}$, then, for all $t \geq 0, h>0$, for all $(x, y) \in C$, and all $\lambda>0$ with $\lambda \omega<1$,

$$
\begin{aligned}
(1-\lambda \omega) & \frac{e^{\omega h}-1}{\omega h}\|u(t)-x\| \\
\leq & \frac{\lambda}{h}\|u(t+h)-u(t)\|+\frac{2}{h} \int_{0}^{h} e^{\omega(h-\tau)}\|u(t+\tau)-u(t)\| d \tau \\
& +\frac{e^{\omega h}-1}{\omega h}\|x+\lambda y-u(t)\|+\frac{\lambda}{h} \int_{0}^{h} e^{\omega(h-\tau)}\|f(t+\tau)\| d \tau
\end{aligned}
$$

(with the convention $\left(e^{r}-1\right) / r=1$ for $\left.r=0\right)$.

Specializing this result to $C=B, \omega=0, f(t)=F\left(\left(u_{\varphi}\right)_{t}\right), x=J_{\lambda}{ }^{B} u \varphi(t), y=$ $\frac{1}{\lambda}\left(I-J_{\lambda}{ }^{B}\right) u_{\varphi}(t) \in B J_{\lambda}{ }^{B} u_{\varphi}(t)$, and $h=\lambda$,

$$
\begin{aligned}
\left\|u_{\varphi}(t)-J_{\lambda}{ }^{B} u_{\varphi}(t)\right\| & \leq\left\|u_{\varphi}(t+\lambda)-u_{\varphi}(t)\right\|+\frac{2}{\lambda} \int_{0}^{\lambda}\left\|u_{\varphi}(t+\tau)-u_{\varphi}(t)\right\| d \tau \\
& +\int_{0}^{\lambda}\left\|F\left(\left(u_{\varphi}\right)_{t+\tau}\right)\right\| d \tau
\end{aligned}
$$

for all $t \geq 0$, and all $\lambda>0$ small enough. Noting that both $u_{\varphi}\left(\mathbb{R}^{+}\right)$and $\left\{F\left(\left(u_{\varphi}\right)_{t}\right) \mid\right.$ $t \geq R\}$ are $L^{\infty}$-bounded (for the latter set, note that by (a), $0 \leq\left(u_{\varphi}\right)_{t}(s) \leq$ $\max \{\|\varphi(0)\|, 1 / b\}$ for all $t \geq R, s \in[-R, 0])$, the assumption on $J_{\lambda}{ }^{B}$ and uniform continuity of $u_{\varphi}$ thus imply that, given any $\epsilon>0$, the set $u_{\varphi}([R, \infty))$ is $\epsilon$-close in the $L^{1}$-norm to an $L^{1}$-relatively compact set, and thus is $L^{1}$-relatively compact as well.

We turn to (c2), (ii): Let $\left(S_{B}(t)\right)_{t \geq 0}$ be the semigroup generated by $-B$. Given $r \geq 0$, (a) $u_{\varphi}(r+\cdot)$ is a mild solution to $\dot{u}(t)+B u(t) \ni F\left(\left(u_{\varphi}\right)_{t+r}\right), u(0)=u_{\varphi}(r)$, 
and (b) $S_{B}(\cdot) u_{\varphi}(r)$ is a mild solution to $\dot{v}(t)+B v(t) \ni 0, v(0)=u_{\varphi}(r)$. Thus,

$$
\left\|u_{\varphi}(t+r)-S_{B}(t) u_{\varphi}(r)\right\| \leq \int_{0}^{t}\left\|F\left(\left(u_{\varphi}\right)_{\tau+r}\right)\right\| d \tau \quad \text { for all } r, t \geq 0 .
$$

Noting, once again, that both $u_{\varphi}\left(\mathbb{R}^{+}\right)$and $\left\{F\left(\left(u_{\varphi}\right)_{t}\right) \mid t \geq R\right\}$ are $L^{\infty}$-bounded, and letting $t>0$ be small enough, and $r \geq R$, the assumption on $\left(S_{B}(t)\right)_{t \geq 0}$ implies that, given any $\epsilon>0$, the set $u_{\varphi}([R+1, \infty))$ is $\epsilon$-close in the $L^{1}$-norm to an $L^{1}$-relatively compact set, and thus is $L^{1}$-relatively compact as well. This completes the proof of (c2).

As (c3) is an obvious consequence of (5.5) and the fact that, for a finite measure space, $L^{\infty}$-order intervals are $L^{1}$-weakly relatively compact, we turn to the proof of (d): If $(B+\omega I)$ is accretive, then, as $(0,0) \in B$, and thus the constant zerofunction is a solution to (5.4), we read from (2.2) that

$$
e^{-\omega t}\left\|u_{\varphi}(t)\right\|-e^{-\omega s}\left\|u_{\varphi}(s)\right\| \leq \int_{s}^{t} e^{-\omega \tau}\left[u_{\varphi}(\tau), F\left(\left(u_{\varphi}\right)_{\tau}\right)\right] d \tau \quad \text { for all } t \geq 0 .
$$

Invoking (5.9) for $\beta=0$,

$$
\begin{aligned}
{\left[u_{\varphi}(\tau), F\left(\left(u_{\varphi}\right)_{\tau}\right)\right]=\int_{(u(\tau)>0)} F\left(\left(u_{\varphi}\right)_{\tau}\right) } & =a \int_{(u(\tau)>0)} u_{\varphi}(\tau)\left(1-b u_{\varphi}(\tau)-G\left(u_{\varphi}\right)_{\tau}\right) \\
& \leq a\left\|u_{\varphi}(\tau)\right\|,
\end{aligned}
$$

we conclude from (5.12) that

$$
e^{-\omega t}\left\|u_{\varphi}(t)\right\| \leq\|\varphi(0)\|+a \int_{0}^{t} e^{-\omega \tau}\left\|u_{\varphi}(\tau)\right\| d \tau \text { for all } t \geq 0
$$

An application of Gronwall's Lemma completes the proof.

Remark 5.6. We note in passing that the above proof of Proposition 5.1 (a) underlines a subtlety in the assumptions on $F$ : for models of the type above, (A4) (c) can be verified, but cannot be strengthened to $\left\|F \varphi_{1}-F \varphi_{2}\right\| \leq M_{F}\left\|\varphi_{1}-\varphi_{2}\right\|$ for those pairs of initial histories.

In closing this paper, we note that, while, incidentally, both models (5.4) and (5.6) include finite delays, the techniques developed so far work just as well for infinite-delay models, and, perhaps more importantly, for temporal averages replaced by spatio-temporal averages over the full past history, such as

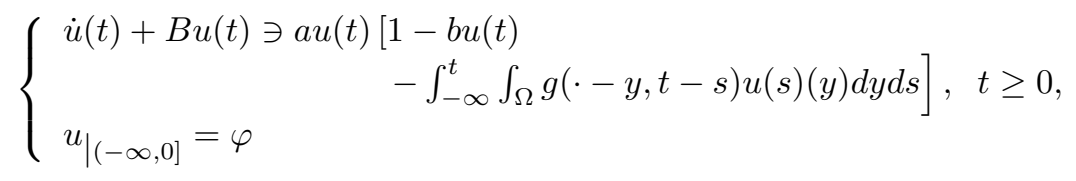

and

$$
\left\{\begin{array}{c}
\dot{u}(t)+B u(t) \ni u(t)\left[1+a u(t)-b(u(t))^{2}\right. \\
\left.\quad-(1+a-b) \int_{-\infty}^{t} \int_{\Omega} g(\cdot-y, t-s) u(s)(y) d y d s\right], t \geq 0, \\
u_{(-\infty, 0]}=\varphi
\end{array}\right.
$$

with $g \in L^{1}\left(\mathbb{R}^{N} \times(0, \infty)\right)$ nonnegative and suitably chosen, and $B$ as in (5.2) and (5.3). (For the discussion of model (5.14) for $B=-\Delta$, together with further relevant references, the reader is referred to [13.) Once again, as for models (5.4) and (5.6), global existence and flow-invariance results corresponding to Proposition 
5.1 can be deduced in the $L^{1}$-context by the above techniques for models (5.13) and (5.14).

\section{ACKNOWLEDGEMENT}

The author is grateful to the referee for various helpful comments. Also, the referee has suggested the following interesting variant of the general regularity problem posed at the end of Step 3 of the proof of Theorem 4.5: consider a $p$ Laplacian type operator $C$ in $L^{1}(\Omega) \cap L^{\infty}(\Omega)$ (such as the operator $A_{0}$ of section 5 above). Then, as $\bar{C}$ in $L^{1}(\Omega)$ is m-completely accretive, condition $H(0,0)$ (of Step 2 of the proof of Theorem 2.1 above) is satisfied for $C$ and $Z=F=L^{1}(\Omega)$. Thus, given $u_{0} \in \operatorname{cl} D(C)$, there exists a unique mild solution to $\dot{u}+C u \ni 0, u(0)=u_{0}$. Moreover, once again due to m-complete accretivity of $\bar{C}$, if $u_{0} \in D(\bar{C})$, then this is actually a strong solution to the corresponding Cauchy problem for $\bar{C}$ (cf. 6 , Thm. 4.2]), and the question is whether it would also be a strong solution for $C$. This would amount to a certain $L^{1}-L^{\infty}$-smoothing effect in this special context.

\section{REFERENCES}

[1] M. Adimy and K. Ezzinbi, Local existence and linearized stability for partial functionaldifferential equations, Dynam. Systems Appl. 7 (1998), 389-403. MR1639596 (99m:34181)

[2] H. Amann, Invariant sets and existence theorems for semilinear parabolic and elliptic systems, J. Math. Anal. Appl. 65 (1978), 432-467. MR506318 (80b:35083)

[3] K. Ammar and P. Wittbold, Quasi-linear parabolic problems in $L^{1}$ with non-homogeneous conditions on the boundary, J. Evol. Equ. 5 (2005), 1-33. MR2125404 (2005m:35139)

[4] F. Andreu, J.M. Mazon, S. Seguera de Leon and J. Toledo, Quasilinear elliptic and parabolic equations in $L^{1}$ with nonlinear boundary conditions, Adv. Math. Sci. Appl. 7 (1997), 183-213. MR:1454663 (98f:35079)

[5] Ph. Bénilan, L. Boccardo, Th. Gallouët, R. Gariepy, M. Pierre and J.L. Vazquez, An $L^{1}$-theory of existence and uniqueness of solutions of nonlinear elliptic equations, Annali Scuola Norm. Sup. Pisa 22 (1995), 241-273. MR.1354907 (96k:35052)

[6] Ph. Bénilan and M.G. Crandall, Completely accretive operators, Semigroup Theory and Evolution Equations (E. Mitidieri, P. Clément and B. de Pagter, eds.), Lecture Notes Pure Appl. Math., no. 135, Marcel Dekker, 1991, pp. 41-75. MR.1164641(93e:47071)

[7] Ph. Bénilan, M.G. Crandall, and A. Pazy, Evolution Equations Governed by Accretive Operators, Springer, in preparation.

[8] Ph. Bénilan and P. Wittbold, Absorptions nonlinéaires, J. Funct. Anal. 114 (1993), 59-96. MR.1220983 (94c:47075)

[9] D. Bothe, Nonlinear Evolutions in Banach Spaces: Existence and qualitative theory with applications to reaction-diffusion systems, Habilitationsschrift, Universität Paderborn, Paderborn, Germany (1999), 160 pp.

[10] D. Bothe, Flow invariance for nonlinear accretive evolutions under range conditions, J. Evol. Equ. 5 (2005), 227-252. MR2133443 (2006b:34140)

[11] D.W. Brewer, A nonlinear semigroup for a functional differential equation, Trans. Amer. Math. Soc. 236 (1978), 173-191. MR0466838 (57:6712)

$[12]$ D.W. Brewer, The asymptotic stability of a nonlinear functional differential equation of infinite delay, Houston J. Math. 6 (1980), 321-330. MR.597773 (81k:34081)

[13] N.F. Britton, Spatial structures and periodic travelling waves in an integro-differential reaction-diffusion population model, SIAM J. Appl. Math. 50 (1990), 1663-1688. MR.1080515 (91m:92020)

[14] C. Corduneanu and V. Lakshmikantham, Equations with unbounded delay: A survey, Nonlinear Analysis TMA 4 (1979), 831-877. MR586852 (81i:34061)

[15] M.G. Crandall, A generalized domain for semigroup generators, Proc. Amer. Math. Soc. 37 (1973), 434-440. MR0313873 (47:2426)

[16] M.G. Crandall and A. Pazy, Nonlinear evolution equations in Banach spaces, Israel J. Math. 11 (1972), 57-94. MR0300166(45:9214) 
[17] G. da Prato and E. Sinestrari, Differential operators with nondense domain, Ann. Scuola Norm. Sup. Pisa Cl. Sci. 14 (1987), 285-344. MR939631 (89f:47062)

[18] W. Desch and W. Schappacher, Linearized stability for nonlinear semigroups. In: Differential Equations in Banach Spaces (A. Favini and E. Obrecht, Eds.), pp. 61-73, Lecture Notes in Math., Vol. 1223, Springer, New York, 1986. MR872517 (88k:47083)

[19] J. Dyson and R. Villella-Bressan, Functional differential equations and non-linear evolution operators, Proc. Royal Soc. Edinburgh 75A (1975/76), 223-234. MR0442402 (56:784)

[20] J. Dyson and R. Villella-Bressan, Semigroups of translations associated with functional and functional differential equations, Proc. Royal Soc. Edinburgh 82A (1979), 171-188. MR532900 (80i:34113)

[21] J. Dyson and R. Villella-Bressan, Nonautonomous locally Lipschitz continuous functional differential equations in spaces of continuous functions, Nonlinear Diff. Eqns. Appl. 3 (1996), 127-147. MR1371098 (96m:34023)

[22] H. Engler, Invariant sets for functional differential equations in Banach spaces and applications, Nonlinear Analysis TMA 5 (1981), 1225-1243. MR636733 (84k:34084)

[23] K. Ezzinbi and A. Ouhinou, Necessary and sufficient conditions for the regularity and stability for some partial functional differential equations with infinite delay, Nonlinear Analysis TMA 64 (2006), 1690-1709. MR2197356 (2006j:34194)

[24] W.E. Fitzgibbon, Nonlinear Volterra equations with infinite delay, Mh. Mathematik 84 (1977), 275-288. MR0481982 (58:2072)

[25] H. Flaschka and M.J. Leitman, On semigroups of nonlinear operators and the solution of the functional differential equation $\dot{x}(t)=F\left(x_{t}\right)$, J. Math. Anal. Appl. 49 (1975), 649-658. MR0361959 (50:14401)

[26] S.M. Ghavidel, Existence and flow-invariance of solutions to non-autonomous partial differential delay equations, Dissertation, Universität Duisburg-Essen, Essen, 2007.

[27] S.A. Gourley and N.F. Britton, On a modified Volterra population equation with diffusion, Nonlinear Analysis TMA 21 (1993), 389-395. MR1237130 (94g:45004)

[28] J.K. Hale, Functional differential equations with infinite delays, J. Math. Anal. Appl. 48 (1974), 276-283. MR0364813 (51:1067)

[29] J.K. Hale, Large diffusivity and asymptotic behavior in parabolic systems, J. Math. Anal. Appl. 118 (1986), 455-466. MR852171 (87i:35101)

[30] J.K. Hale and J. Kato, Phase space for retarded equations with infinite delay, Funkcial. Ekvac. 21 (1978), 11-41. MR0492721 (58:11793)

[31] J.K. Hale and S.M. Verduyn Lunel, Introduction to Functional Differential Equations, Appl. Math. Sci., no. 99, Springer, New York, 1993.

[32] F. Kappel and W. Schappacher, Some considerations to the fundamental theory of infinite delay equations, J. Diff. Eqns. 37 (1980), 141-183. MR587220 (81j:34117)

[33] A.G. Kartsatos, A direct method for the existence of evolution operators associated with functional evolutions in general Banach spaces, Funkcial. Ekvac. 31 (1988), 89-102. MR951768 (89j:34093)

[34] A.G. Kartsatos and M.E. Parrott, Global solutions of functional evolution equations involving locally defined Lipschitzian perturbations, J. London Math. Soc. (2) 27 (1983), 306-316. MR692536 (85g:34066)

[35] A.G. Kartsatos and M.E. Parrott, The weak solution of a functional differential equation in a general Banach space, J. Diff. Eqns. 75 (1988), 290-302. MR961157 (89k:34134)

[36] N. Kato, A principle of linearized stability for nonlinear evolution equations, Trans. Amer. Math. Soc. 347 (1995), 2851-2868. MR.1290722 (95j:34091)

[37] K. Kunisch and W. Schappacher, Mild and strong solutions for partial differential equations with delay, Annali Mat. Pura Appl. Ser. 4125 (1980), 193-219. MR605209 (82i:34100)

[38] K. Kunisch and W. Schappacher, Variation of constants formulas for partial differential equations with delay, Nonlinear Analysis TMA 5 (1981), 123-142. MR606694 (82i:35162)

[39] V. Lakshmikhantam, S. Leela and V. Moauro, Existence and uniqueness of solutions of delay differential equations on a closed subset of a Banach space, Nonlinear Analysis TMA 2 (1978), 311-327. MR.512662 (80c:34069)

[40] S. Leela and V. Moauro, Existence of solutions in a closed set for delay differential equations in Banach spaces, Nonlinear Analysis TMA 2 (1978), 47-58. MR.512653 (80c:34070)

[41] J.H. Lightbourne III, Function space flow-invariance for functional differential equations of retarded type, Proc. Amer. Math. Soc. 77 (1979), 91-98. MR539637 (80j:34089) 
[42] R.H. Martin, Nonlinear Operators and Differential Equations in Banach Spaces, Wiley, New York, 1976. MR0492671 (58:11753)

[43] R.H. Martin and H.L. Smith, Abstract functional differential equations and reaction-diffusion systems, Trans. Amer. Math. Soc. 321 (1990), 1-44. MR967316 (90m:35194)

[44] R.H. Martin and H.L. Smith, Convergence in Lotka-Volterra systems with diffusion and delay, Differential Equations with Applications in Biology, Physics and Engineering (J.A. Goldstein, F. Kappel, and W. Schappacher, eds.), Lecture Notes Pure Appl. Math., no. 133, Marcel Dekker, 1991, pp. 259-267. MR.1171475

[45] R.H. Martin and H.L. Smith, Reaction-diffusion systems with time delays: Monotonicity, invariance, comparison and convergence, J. reine angew. Math. 413 (1991), 1-35. MR.1089794 (92b:35162)

[46] M.C. Memory, Invariant manifolds for partial functional differential equations, Proc. 2nd Int. Conf. Math. Population Dynamics, 1991, pp. 223-232. MR.1227366 (94g:35214)

[47] M.C. Memory, Stable and unstable manifolds for partial functional differential equations, Nonlinear Analysis TMA 16 (1991), 131-142. MR1090786 (92a:35156)

[48] I. Miyadera, Nonlinear Semigroups, Transl. of Math. Monographs, no. 109, Amer. Math. Soc., Providence, RI, 1992. MR1192132 (93j:47093)

[49] S. Murakami, Stable equilibrium point of some diffusive functional differential equations, Nonlinear Analysis TMA 25 (1995), 1037-1043. MR1350725 (96j:35259)

[50] R. Nagel et al., One-parameter Semigroups of Positive Operators, Lecture Notes Math., no. 1184, Springer, Berlin, 1986. MR0839450 (88i:47022)

[51] M.E. Parrott, Representation and approximation of generalized solutions of a nonlinear functional differential equation, Nonlinear Analysis TMA 6 (1982), 307-318. MR654808 (83d:34127)

[52] M.E. Parrott, Positivity and a principle of linearized stability for delay-differential equations, Diff. Integral Eqns. 2 (1989), 170-182. MR.984185 (90b:34110)

[53] M.E. Parrott, Linearized stability and irreducibility for a functional differential equation, SIAM J. Math. Anal. 23 (1992), 649-661. MR.1158826 (93f:34134)

[54] N.H. Pavel, Invariant sets for a class of semilinear equations of evolution, Nonlinear Analysis TMA 1 (1977), 187-196.

[55] N. Pavel and F. Iacob, Invariant sets for a class of perturbed differential equations of retarded type, Israel J. Math. 28 (1977), 254-264. MR0466829 (57:6703)

[56] N.H. Pavel, Differential equations, flow invariance and applications, Research Notes Math. 113, Pitman, Boston, London, Melbourne 1984. MR774954 (86g:58027)

[57] M. Pierre, Invariant closed subsets for nonlinear semigroups, Nonlinear Analysis TMA 2 (1978), 107-117. MR512659 (80b:47093)

[58] A.T. Plant, Nonlinear semigroups of translations in Banach space generated by functional differential equations, J. Math. Anal. Appl. 60 (1977), 67-74. MR0447745|(56:6055)

[59] A.T. Plant, Stability of nonlinear functional differential equations using weighted norms, Houston J. Math. 3 (1977), 99-108. MR0430478 (55:3483)

[60] J. Prüss, On semilinear parabolic equations on closed sets, J. Math. Anal. Appl. 77 (1980), 513-538. MR593232 (82c:34076)

[61] R. Redlinger, On Volterra's population equation with diffusion, SIAM J. Math. Anal. 16 (1985), 135-142. MR772872 (86c:92025)

[62] W.M. Ruess, The evolution operator approach to functional differential equations with delay, Proc. Amer. Math. Soc. 119 (1993), 783-791. MR.1154248 (93m:34132)

[63] W.M. Ruess, Compactness and asymptotic stabilty for solutions of functional differential equations with infinite delay, Evolution Equations (G. Ferreyra, G. Ruiz Goldstein, and F. Neubrander, eds.), Lecture Notes Pure Appl. Math., no. 168, Marcel Dekker, 1994, pp. 361374. MR1300442 (96a:34153)

[64] W.M. Ruess, Asymptotic behavior of solutions to delay functional differential equations, Recent Developments in Evolution Equations (A.C. McBride and G.F. Roach, eds.), Pitman Research Notes Math., no. 324, Longman, 1995, pp. 220-230. MR1417077 (97i:34110)

[65] W.M. Ruess, Existence of solutions to partial functional differential equations with delay, Theory and Applications of Nonlinear Operators of Accretive and Monotone Type (A.G. Kartsatos, ed.), Lecture Notes Pure Appl. Math., no. 178, Marcel Dekker, 1996, pp. 259-288. MR1386683(97b:34085) 
[66] W.M. Ruess, Existence of solutions to partial functional evolution equations with delay, Functional Analysis (S. Dierolf, S. Dineen and P. Domanski, Eds.), de Gruyter 1996, 377-387. MR.1420463 (97j:34103)

[67] W.M. Ruess, Existence and stability of solutions to partial functional differential equations with delay, Adv. Diff. Eqns. 4 (1999), 843-876. MR.1729393 (2000m:34184)

[68] W.M. Ruess, Linearized stability for nonlinear evolution equations, J. Evol. Equ. 3 (2003), 361-373. MR.1980982 (2005h:34165)

[69] W.M. Ruess, Linearized stability and regularity for nonlinear age-dependent population models, Functional Analysis and Evolution Equations. The Günter Lumer Volume (H. Amann, W. Arendt, M. Hieber, F. Neubrander, S. Nicaise, J. von Below, eds.), Birkhäuser, Basel 2007, 561-576.

[70] W.M. Ruess and W.H. Summers, Operator semigroups for functional differential equations with delay, Trans. Amer. Math. Soc. 341 (1994), 695-719. MR1214785 (94g:34135)

[71] W.M. Ruess and W.H. Summers, Almost periodicity and stability for solutions to functional differential equations with delay, Diff. Integral Eqns. 9 (1996), 1225-1252. MR 1409925 (97m:34159)

[72] W.M. Ruess and W.H. Summers, Linearized stability for abstract differential equations with delay, J. Math. Anal. Appl. 198 (1996), 310-336. MR1376267 (97a:34208)

[73] K. Sbihi, B. Andreianov and P. Wittbold, On uniqueness and existence of entropy solutions for a nonlinear parabolic problem with absorption, submitted.

[74] A. Schiaffino, On a diffusion Volterra equation, Nonlinear Analysis TMA 3 (1979), 595-600. MR.541870 (80h:35077)

[75] G. Seifert, Positively invariant closed sets for systems of delay differential equations, J. Diff. Eqns. 22 (1976), 292-304. MR0427781 (55:811)

[76] C.C. Travis and G.F. Webb, Existence and stability for partial functional differential equations, Trans. Amer. Math. Soc. 200 (1974), 395-418. MR0382808 (52:3690)

[77] C.C. Travis and G.F. Webb, Partial differential equations with deviating arguments in the time variable, J. Math. Anal. Appl. 56 (1976), 397-409. MR0415102 (54:3193)

[78] I.I. Vrabie, Compactness Methods for Nonlinear Evolutions, Pitman Monographs and Surveys Pure Appl. Math. 32, Longman, New York, 1987. MR932730 (90f:47101)

[79] G.F. Webb, Autonomous nonlinear functional differential equations and nonlinear semigroups, J. Math. Anal. Appl. 46 (1974), 1-12. MR0348224 (50:722)

[80] G.F. Webb, Asymptotic stability for abstract nonlinear functional differential equations, Proc. Amer. Math. Soc. 54 (1976), 225-230. MR0402237 (53:6058)

[81] G.F. Webb, Volterra integral equations and nonlinear semigroups, Nonlinear Analysis TMA 1 (1977), 415-427. MR.511684 (80b:45005)

[82] G.F. Webb, Volterra integral equations as functional differential equations on infinite intervals, Hiroshima J. Math. 7 (1977), 61-70. MR0445247 (56:3591)

[83] P. Wittbold, Absorptions nonlinéaires, Thèse doctorat, Université de Besançon, Besançon, France, 1994.

[84] P. Wittbold, Diffusion nonlinéaire avec absorption, C.R. Acad. Sci. Paris 319, Série I (1994), 675-678. MR.1300068 (95g:35221)

[85] P. Wittbold, Nonlinear diffusion with absorption, Progress in partial differential equations: the Metz surveys 4 (M. Chipot and I. Shafrir, eds.), Pitman Res. Notes Math. Series, no. 345, Longman, 1996, pp. 142-157. MR.1394711 (97c:35110)

[86] P. Wittbold, Nonlinear diffusion with absorption, Potential Analysis 7 (1997), 437-465. MR.1462580 (98f:35058)

[87] J. Wu, Theory and Applications of Partial Functional Differential Equations, Appl. Math. Sci., no. 119, Springer, New York, 1996. MR.1415838 (98a:35135)

[88] K. Yoshida, The Hopf bifurcation and its stability for semilinear diffusion equations with time delay arising in ecology, Hiroshima Math. J. 12 (1982), 321-348. MR665499 (83m:58066)

Fachbereich Mathematik, Universität Duisburg-Essen, D-45117 Essen, Germany

E-mail address: wolfgang.ruess@uni-due.de 